\title{
Classical Limits for Quantum Particles in External Yang-Mills Potentials
}

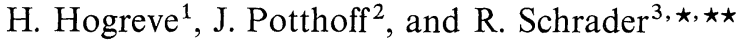 \\ 1 Institut für Theoretische Physik, Freie Universität, D-1000 Berlin \\ 2 Fakultät für Physik, Universität Bielefeld, D-4800 Bielefeld, Federal Republic of Germany \\ 3 Institute for Theoretical Physics, SUNY at Stony Brook, Stony Brook, NY 11794, USA
}

\begin{abstract}
We consider the limit $\hbar \rightarrow 0$ for nonrelativistic quantum particles moving in external Yang-Mills potentials. It is shown that the partition function and the solutions of the equations of motion converge to their corresponding classical counterparts.
\end{abstract}

\section{Introduction}

The equations governing the motion of a classical particle moving in an external SU(2) Yang-Mills potential were first obtained by Wong [Wo] by taking the formal limit $\hbar \rightarrow 0$ of the quantum mechanical equations of motion. Since then, various aspects of these equations have been studied and used [BSSW, BCL, BW, $\mathrm{DC}, \mathrm{Sch}, \mathrm{S}]$, as well as extended [GS, St] to arbitrary groups using the symplectic structure of coadjoint orbits $[\mathrm{Ki}, \mathrm{Ko}$, Sou]. We will review this classical formulation in Sect. 2 in a form suited for the applications we have in mind.

The principle of minimal coupling leads to a prescription of how to couple a quantum mechanical particle with internal degrees of freedom, like isospin in the $\mathrm{SU}(2)$ case, to an external Yang-Mills potential. In mathematical language, minimal coupling amounts to the replacement of the ordinary Laplace operator by the Laplace-Bochner operator obtained from the connection, whose Christoffel symbols just form the given Yang-Mills vector potential. This Laplace-Bochner operator thus describes the interaction with the "magnetic" part of the YangMills potential and is of interest in quantum field theory, because in its euclidean formulation it describes the coupling of Higg's fields to Yang-Mills fields.

In addition the Hamiltonian may contain a scalar (with respect to space-time) Yang-Mills potential, describing an "electric" interaction. We present this setup in Sect. 3 together with some concepts from group theory needed in this context.

In the theory of quantum statistical spin systems it is well known that in order to obtain the corresponding classical theory when $\hbar \rightarrow 0$, it is necessary to let the

* Supported in part by Deutsche Forschungsgemeinschaft and NSF Grant No. PHY 81-09110 A-01

$\star \star$ On leave of absence from Freie Universität, D-1000 Berlin 
representation of the internal symmetry [i.e. the isospin, when the gauge group is $\mathrm{SU}(2)]$ tend to infinity like $\hbar^{-1}$. Rescaling the Yang-Mills fields by $\hbar$ will then give a gauge-covariant formulation, which does not contain $\hbar$ explicitly. Therefore gauge covariance will also hold in the limit.

In Sect. 5, we will show that the quantum mechanical Gibbs partition function tends to the corresponding classical partition function. In particular, as for the electromagnetic [i.e. U(1)] case [CSS], the "magnetic" dependence drops out in the limit, so classically there is also no para- or diamagnetism in the noncommutative case. The "electric" part on the other hand we will control using classical limit theorems on quantum spin observables in a form first obtained by Gilmore [G] and then extended by Simon [Si1]. The method of proof also employs the stochastic, noncommutative integral formulation for Yang-Mills potentials to write the kernel of the semigroup of the Laplace-Bochner operator in terms of the Brownian motion. We combine this with the concept of the Brownian bridge, so our proof is an adaptation of the one given by Simon [Si2] for the $\hbar \rightarrow 0$ limit in the electromagnetic case.

Hepp's version [H] of the Ehrenfest formulation [E] of the $\hbar \rightarrow 0$ limit for the equations of motion is powerful enough to be extended to the present situation. This discussion will be given in Sect. 6. The new input again consists in using coherent states on Lie groups as given by Gilmore and Simon and already referred to (see also [K1, Pe]). Note that in Hepp's discussion, the procedure of taking the $\hbar \rightarrow 0$ limit is compatible with the time evolution in the quantum mechanical and classical case respectively. Technically, this was achieved by using suitable Weyl transformations. In the present situation, we use suitable gauge transformations in addition in order to control the behaviour in the internal symmetry space.

Finally, as in Hepp's discussion we obtain a description of the quantum corrections to the classical equations of motion by linearizing the non-linear Heisenberg equations of motion around the classical orbit. In particular this leads to a description of quantum corrections to the classical motion in the internal symmetry space. The necessary group theoretical structures are presented in Sect. 4.

\section{The Classical Theory}

In this section we give a brief review of the classical theory of a particle moving in an external Yang-Mills potential as described by the Wong equations [Wo]. Let $G$ be a compact Lie group with a discrete center (三semisimple), $g$ its (real) Lie algebra and $g^{*}$ the real dual of $g$. By duality the adjoint representation $\operatorname{Ad}(\cdot)$ of $G$ on $g$ induces a representation $\mathrm{Ad}^{*}(\cdot)$ of $G$ on $g^{*}$, called the coadjoint representation, by $\quad \operatorname{Ad}^{*}(g)=\operatorname{Ad}\left(g^{-1}\right)^{*}$.

Orbits of $\mathrm{Ad}^{*}(\cdot)$ are called coadjoint orbits. By definition they are of the form

$$
\Gamma=\left\{l \in g^{*} \mid l=\operatorname{Ad}^{*}(g) l_{0}, g \in G\right\}
$$

for a fixed $l_{0}$ in $g^{*}$, i.e. $\Gamma$ is the orbit through $l_{0}$. We define the normalized measure $\mu_{\Gamma}$ on $\Gamma$ as the pullback of the normalized Haar measure $\mu$ on $G$ :

$$
\mu_{\Gamma}(B)=\mu\left\{g \in G \mid \operatorname{Ad}^{*}(g) l_{0} \in B\right\} .
$$


$\Gamma$ is a symplectic manifold, the symplectic structure being given as follows (see e.g. $[\mathrm{Ki}, \mathrm{Ko}, \mathrm{Sou}])$. The cotangent space $T_{l}^{*}(\Gamma)$ at $l \in \Gamma$ is naturally associated to a quotient space of $\left(g^{*}\right)^{*} \cong g$ by

$$
T_{l}^{*}(\Gamma) \cong g /\{a \in g / l([a, b])=0 \text { for all } b \in g\},
$$

with [, ] denoting the commutator in $g$. If $\{a\}\left\{a^{\prime}\right\}\left(a, a^{\prime} \in g\right)$ are any two elements in this space, then the skew symmetric quadratic form on $T_{l}^{*}(\Gamma)$,

$$
\omega_{l}\left(\{a\},\left\{a^{\prime}\right\}\right)=l\left(\left[a, a^{\prime}\right]\right),
$$

is well defined, nondegenerate and closed.

Let $A_{\mu}(x, t)\left(\mu=0, \ldots, v, x \in \mathbb{R}^{v}, t \in \mathbb{R}\right)$ be $g$-valued functions on $\mathbb{R}^{v} \times \mathbb{R}$, which for simplicity we assume to be in $C_{0}^{\infty}$. In the phase space $T^{*} \mathbb{R}^{v} \times \Gamma \cong \mathbb{R}^{2 v} \times \Gamma(\Gamma$ a fixed coadjoint orbit) with points in it denoted by $(x, p, l)=\left(x_{1}, \ldots, x_{v}, p_{1}, \ldots, p_{v}, l\right)$ consider the time dependent Hamiltonian

$$
H(x, p, l, t)=\frac{1}{2 m} \sum_{j=1}^{v}\left(p_{j}+l\left(A_{j}(x, t)\right)^{2}+l\left(A_{0}(x, t)\right)+V(x),\right.
$$

where $V(x)$ is a real valued $C^{\infty}$ function, whose further properties will be specified below. With the symplectic structure on $T^{*} \mathbb{R}^{v} \times \Gamma$, this Hamiltonian gives the following Hamiltonian equations

$$
\begin{gathered}
m \dot{x}_{j}=p_{j}+l\left(A_{j}(x, t)\right), \\
\left.\dot{p}_{j}=-\frac{1}{m}\left(p_{k}+l\left(A_{k}(x, t)\right)\right)\right)\left(\partial_{j} A_{k}(x, t)\right)-l\left(\partial_{j} A_{0}(x, t)\right)-\partial_{j} V, \\
\dot{l}(a)=l\left(\left[A_{j}(x, t), a\right]\right) \frac{1}{m}\left(p_{j}+l\left(A_{j}(x, t)\right)\right)+l\left(\left[A_{0}(x, t), a\right]\right),
\end{gathered}
$$

$(a \in g)$ and we have adopted the Einstein summation convention. Equations (2.7) and (2.8) are often referred to as the first and second Wong equations, respectively.

Let now $g(x, t)$ be a $C^{\infty} G$-valued function on $\mathbb{R}^{v} \times \mathbb{R}$ and consider the transformation on $T^{*} \mathbb{R}^{v} \times \Gamma$ given as

$$
(x, p, l) \mapsto\left(x, p-l\left(g(x, t) \partial g^{-1}(x, t)\right), \operatorname{Ad}^{*} g(x, t) l\right),
$$

and the gauge transformation

with

$$
A_{\mu} \mapsto^{g(\cdot)} A_{\mu} \quad(\mu=0, \ldots, v),
$$

$$
{ }^{g(\cdot)} A_{\mu}(x, t)=\operatorname{Ad} g(x, t) A_{\mu}(x, t)+g(x, t) \partial_{\mu} g^{-1}(x, t), \quad\left(\partial_{0}=\partial_{t}\right) .
$$

As usual we view $g(x, t) \partial_{\mu} g^{-1}(x, t)$ as an element of the Lie algebra [since $\partial_{\mu} g^{-1}(x, t) \in T_{g^{-1}(x, t)} G$, and $g(x, t)$ induces a map of $T_{g^{-1}(x, t)} G$ onto $\left.T_{e} G=g\right]$. With this notational convention we also have

$$
\operatorname{Ad} g(x, t) \partial_{\mu} \operatorname{Ad} g^{-1}(x, t)=\operatorname{ad}\left(g(x, t) \partial_{\mu} g^{-1}(x, t)\right) .
$$

It is an easy exercise to see that the equations of motion (2.7) are gauge covariant in the sense that their form is preserved under the simultaneous transformations 
(2.9) and (2.10). Of course this follows also from the invariance of both the symplectic structure and the Hamiltonian under such transformations.

The Eqs. (2.7) in particular lead to the Lorentz equations in the form

$$
m \ddot{x}_{j}=l\left(F_{k j}(x, t)\right) \dot{x}_{k}+l\left(F_{0 j}(x, t)\right)-\partial_{j} V,
$$

with

$$
F_{\mu \tau}=\partial_{\mu} A_{\tau}-\partial_{\tau} A_{\mu}+\left[A_{\mu}, A_{\tau}\right] .
$$

Since the coefficients in the first order Eqs. (2.7) and (2.8) are smooth, to any initial condition $(\xi, \pi, \lambda)$, there is $T>0$ and a smooth solution $\left(\xi^{t}, \pi^{t}, \lambda^{t}\right)$ of these equations for $|t|<T$ with initial data $(\xi, \pi, \lambda)$. For the applications we have in mind it will be convenient to express this local solution in a different form. For this purpose we replace Eqs. (2.7) and (2.8) by the following equations on $\mathbb{R}^{2 v} \times G \ni(x, p, g)(\lambda \in \Gamma$ fixed):

$$
\begin{aligned}
m \dot{x}_{j}= & p_{j}+\lambda\left(\operatorname{Ad} g^{-1} A_{j}(x, t)\right), \\
\dot{p}_{j}= & -\frac{1}{m}\left(p_{k}+\lambda\left(\operatorname{Ad} g^{-1} A_{k}(x, t)\right)\right) \cdot \lambda\left(\operatorname{Ad}^{-1} \partial_{j} A_{k}(x, t)\right) \\
& \quad-\lambda\left(\operatorname{Ad} g^{-1} \partial_{j} A_{0}(x, t)\right)-\partial_{j} V(x), \\
g \partial_{t} g^{-1}= & \frac{1}{m} A_{k}(x, t)\left(p_{k}+\lambda\left(\operatorname{Ad} g^{-1} A_{k}(x, t)\right)\right)+A_{0}(x, t) .
\end{aligned}
$$

If $\left(\xi^{t}, \pi^{t}, \gamma^{t}\right) \in T^{*} \mathbb{R}^{v} \times G(|t|<T)$ is a solution of Eqs. (2.7') and $\left(2.8^{\prime}\right)$ with initial conditions $(\xi, \pi, e)$, then $\left(\xi^{t}, \pi^{t}, \lambda^{t}=\mathrm{Ad}^{*} \gamma^{t} \lambda\right)$ is a solution of (2.7) and (2.8) with initial conditions $(\xi, \pi, \lambda)$. It is possible to formulate a converse statement which, however, we shall not need.

\section{The Quantum Theory}

In this section, we give the quantum mechanical formulation for nonrelativistic particles moving in an external Yang-Mills potential. For simplicity, we will restrict attention to the case where the $A_{\mu}$ are time independent, although it is possible to extend our discussion to the general case.

Let $\pi$ be an irreducible, unitary representation of $G$ with (complex) Hilbert space $h_{\pi}$. We will also use the symbol $\pi$ to denote the resulting representation of the Lie-algebra $g$ as well as its complexification $g_{c}$. By $\mathscr{H}_{\pi}=L^{2}\left(\mathbb{R}^{v}, d^{v} x, h_{\pi}\right)$ $\cong L^{2}\left(\mathbb{R}^{v}, d^{v} x\right) \otimes h_{\pi}$, we denote the Hilbert space of all square integrable functions on $\mathbb{R}^{v}$ with values in $h_{\pi}$. For given $A_{\mu}$, consider the operator

$$
H_{\pi, \hbar}^{1}=-\frac{\hbar^{2}}{2 m}\left(\partial_{j}+\pi\left(A_{j}(x)\right)^{2}-i \hbar \pi\left(A_{0}(x)\right)+V(x)\right.
$$

on $\mathscr{H}_{\pi}$. This will be the operator we will consider in the context of taking the classical limit of the Gibbs quantum partition function

$$
Z_{\beta, \pi, \hbar}=\left(\operatorname{dim} h_{\pi}\right)^{-1} \operatorname{Trace}_{\mathscr{H}_{\pi}} \exp -\beta H_{\pi, \hbar}^{1} .
$$


To guarantee that $Z_{\beta, \pi, \hbar}$ is finite for all $\beta$, we will assume that

$$
\int \exp -\beta V(x) d^{v} x<\infty
$$

for all $\beta>0$. Then the Golden-Thomson-Symanzik inequality [Go, T1, T2, Sy] and the Kato-Simon inequality [Ka, Si3] for Yang-Mills potentials [HSU] easily combine to give the classical bound in the form

$$
\hbar^{v} Z_{\beta, \pi, \hbar} \leqq(2 \pi)^{-v} \exp \left\{\beta \hbar \sup _{x} \| \pi\left(A_{0}(x) \|\right\} \exp -\beta\left(\frac{p^{2}}{2 m}+V(x)\right) d^{v} x d^{v} p .\right.
$$

To discuss the $\hbar \rightarrow 0$ limit for the equations of motion, we start by inserting $\hbar$ symmetrically into the quantum mechanical $p$ and $q$ observables by defining (see $[\mathrm{H}])$

$$
H_{\pi, \hbar}^{2}=-\frac{1}{2 m}\left(\hbar^{1 / 2} \partial_{j}+\hbar \pi\left(A_{j}\left(\hbar^{1 / 2} x\right)\right)\right)^{2}-i \hbar \pi\left(A_{0}\left(\hbar^{1 / 2} x\right)\right)+V\left(\hbar^{1 / 2} x\right) .
$$

Note that $H_{\pi, \hbar}^{1}$ and $H_{\pi, \hbar}^{2}$ are related by the unitary scale transformation

$$
(T(\hbar) \psi)(x)=\hbar^{v / 4} \psi\left(\hbar^{1 / 2} x\right) .
$$

Indeed, we have

$$
T(\hbar) H_{\pi, \hbar}^{1} T(\hbar)^{-1}=H_{\pi, \hbar}^{2} .
$$

For a time independent $G$-valued function $g(\cdot)$ on $\mathbb{R}^{v}$, set

$$
(\pi(g(\cdot)) \psi)(x)=\pi(g(x)) \psi(x)
$$

for $\psi \in \mathscr{H}_{\pi}$. As before, let ${ }^{g(\cdot)} A_{\mu}$ be given by (2.11). Note the $\hbar$-independence of this prescription.

If we write out explicitly the $A_{\mu}$ dependence of the Hamiltonians, we have gauge covariance in the form

$$
\pi(g(\cdot)) H_{\pi, h}^{i}\left(A_{\mu}(\cdot)\right) \pi^{-1}(g(\cdot))=H_{\pi, h}^{i}\left({ }^{g(\cdot)} A_{\mu}(\cdot)\right), \quad i=1,2 .
$$

In particular, the (equal) spectra of $H_{\pi, \hbar}^{i}\left(A_{\mu}(\cdot)\right)$ are gauge invariant and thus also the particular function $Z_{\beta, \pi, h}$. We note that a similar property pertains to the resulting scattering matrix $[\mathrm{CKS}]$ for a large class of $A_{\mu}$ and $V^{\prime} s$.

\section{Coherent States and Classical Limits on Lie Groups}

In order to perform the classical limit, we will let the representation vary with $\hbar$. This section contains the relevant group theoretical properties needed, some of which appear to be new. They will be applied in Sect. 5 and 6 .

First choose a Cartan subalgebra $c$ of $g$. Let $\hat{l}_{0}$ be a fixed fundamental weight (see e.g. [Si1] for a brief exposition of this and related concepts we shall use). For each natural number $n$, let $\pi_{n}$ be the irreducible, unitary representation of $G$ with maximal weight $n \hat{l}_{0}$ and (normalized) weight vector $\psi_{n \hat{l}_{0}}$ in the representation space $h_{n}=h_{\pi_{n}}$ of dimension $d_{n} \cdot \psi_{n \hat{l}_{0}}$ may be chosen to be the vector $\psi_{\hat{l}_{0}} \otimes \ldots \otimes \psi_{\hat{l}_{0}}(n$ factors $)$ in the $n$-fold tensor product of $h_{1}$, where $\psi_{\hat{l}_{0}}$ is the weight vector of maximal weight $\hat{l}_{0}$. 
Then $h_{n}$ is the subspace obtained by applying $\otimes^{n} \pi_{1}(g)(g \in G)$ to $\psi_{n \hat{l}_{0}}$. With this identification, $\pi_{n}$ is the restriction of $\bigotimes^{n} \pi_{1}$ to $h_{n}$. The relation [G, Si1]

$$
\left\langle\psi_{n \hat{l}_{0}}\left|\exp \pi_{n}(a)\right| \psi_{n \hat{l}_{0}}\right\rangle=\left\langle\psi_{\hat{l}_{0}}\left|\exp \pi_{1}(a)\right| \psi_{\hat{l}_{0}}\right\rangle^{n}, \quad a \in G
$$

will be crucial in our discussion.

Since every element of $g_{c}$ may be mapped into $c_{c}$, the complexification of $c$, by some Adg, we have

$$
\left\|\pi_{n}(a)\right\| \leqq n c\|a\|, \quad a \in g_{c}
$$

with a fixed norm on $g_{c}$.

By $\hat{l}_{0}$, we will also denote the element of $g_{c}^{*}$ with the properties

$$
\begin{gathered}
\hat{l}_{0}(a) \psi_{\hat{l}_{0}}=\pi_{1}(a) \psi_{\hat{l}_{0}}, \quad a \in c, \\
\hat{l}_{0}(a)=0, \quad a \in c^{\perp},
\end{gathered}
$$

where ${ }^{\perp}$ denotes the orthogonal complement with respect to the Killing form. Equivalently $\hat{l}_{0}(\cdot)$ is given as [Si1]

$$
\hat{l}_{0}(a)=\left\langle\psi_{\hat{l}_{0}}\left|\pi_{1}(a)\right| \psi_{\hat{l}_{0}}\right\rangle, \quad a \in g_{c} .
$$

Note that $\hat{l}_{0}$ is either zero or purely imaginary on $g$. We let $l_{0}=-i \hat{l}_{0}$ and define $\hat{\Gamma}$ to be the coadjoint orbit through $\hat{l}_{0}$ in $g_{c}$, such that $\Gamma=-i \hat{\Gamma}$ is the coadjoint orbit through $l_{0}$. In the beautiful results of classical limits for quantum spin systems [FL, Li, Si1], the main observation is to use the representations in the combination $n^{-1} \pi_{n}(\cdot)$ when letting $n$ tend to infinity. We will also employ this procedure. Moreover, by our definition of the quantum mechanical Hamiltonians, we are thus led to the following relation between $\hbar$ and $n$ :

$$
n \hbar=1 \text {. }
$$

As a consequence, in our applications the representations will always show up in the form $\hbar \pi_{n}(\cdot)$ with a fixed but arbitrary $\pi_{1}$. Note that in abelian case, $\hbar \pi_{n}(\cdot)$ is independent of $n$, if Relation (4.5) is satisfied and equals $\pi_{1}(\cdot)$ (up to an isomorphism).

To describe the $n \rightarrow \infty$ limit, we introduce coherent vectors in the form emphasized by Gilmore [G] and applied by Simon [Si1]. Let $P_{n}(e)$ be the projection onto the space spanned by the vector $\psi_{n \hat{l}_{0}}$ and for any $g \in G$, consider the one-dimensional projections

$$
P_{n}(g)=\pi_{n}(g) P_{n}(e) \pi_{n}(g)^{-1}
$$

If we let $\hat{l}=\operatorname{Ad}^{*} g \hat{l}_{0}$, then $P_{n}(g)$ depends on $\hat{l}$ only, so we may set $P_{n}(\hat{l})=P_{n}(g)$ and the following completeness relation holds [Si1]:

$$
d_{n} \int P_{n}(\hat{l}) d \mu_{\hat{\Gamma}}(\hat{l})=1_{h_{n}} .
$$

The states defined by these projections are called coherent states. The first part of the following lemma is due to Gilmore [G]. 
Lemma 4.1. For any $a \in \mathscr{g}_{c}, \hat{l} \in \hat{\Gamma}$

$$
\lim _{n \rightarrow \infty} \operatorname{trace}_{h_{n}} P_{n}(\hat{l}) e^{\pi_{n}\left(\frac{a}{n}\right)}=e^{\hat{\imath}(a)} .
$$

Moreover, there is a constant $c$, depending on $\Gamma$ only, such that

$$
\left|\operatorname{trace}_{h_{n}} P_{n}(\hat{l})\left(e^{\pi_{n}\left(\frac{a}{n}\right)}-e^{\hat{\imath}(a)}\right)\right| \leqq c n^{-1 / 2}\|a\|(\|\hat{l}\|+c)^{e\|\| a \|(\|\hat{l}\|+c)} .
$$

Proof. It suffices to prove the bound (4.9), so using estimate (4.2) and Schwarz inequality, we estimate the left hand side as $\left(\hat{l}=\mathrm{Ad}^{*} g \hat{l}_{0}\right)$

$$
\begin{aligned}
& \left|\int_{0}^{1} \operatorname{trace}_{h_{n}} P_{n}(\hat{l}) e^{s \pi_{n}\left(\frac{a}{n}\right)+(1-s) \hat{l}(a)}\left(\pi_{n}\left(\frac{a}{n}\right)-\hat{l}(a)\right) d s\right| \\
& \leqq e^{\left\|\pi_{n}\left(\frac{a}{n}\right)\right\|+|\hat{l}(a)|} \operatorname{trace}_{h_{n}} P_{n}(\hat{l})\left(\pi_{n}\left(\frac{a}{n}\right)-\hat{l}(a)\right)\left(\pi_{n}\left(\frac{a}{n}\right)-\hat{l}(a)\right)^{*} \\
& \left.\leqq e^{\|a\|(c+\mid \hat{l} \|)}\right) \operatorname{trace}_{h_{n}} P_{n}\left(\hat{l}_{0}\right) \\
& \quad \cdot\left(\pi_{n}\left(\operatorname{Ad}^{-1} \frac{a}{n}\right)-\hat{l}_{0}\left(\operatorname{Ad} g^{-1} a\right)\right)\left(\pi_{n}\left(\operatorname{Ad}^{-1} \frac{a}{n}\right)-\hat{l}_{0}\left(\operatorname{Ad}^{-1} a\right)\right)^{*} .
\end{aligned}
$$

Now for $b \in g_{c}$

$$
\pi_{n}(b) \psi_{n \hat{l}_{0}}=\sum_{j=1}^{n} \psi_{\hat{l}_{0}} \otimes \ldots \otimes \pi_{1}(b) \psi_{\hat{l}_{0}} \otimes \ldots \otimes \psi_{\hat{l}_{0}},
$$

where in the $j^{\text {th }}$ summand $\pi_{1}(b)$ acts on the $j^{\text {th }}$ factor. Inserting this into estimate (4.10) allows us to rewrite this estimate as

$$
\left(\left(\frac{n(n-1)}{n^{2}}-1\right)|\hat{l}(a)|^{2}+\frac{n}{n^{2}} \| \pi_{1}\left(\operatorname{Ad} g^{-1} a\right)\left|\psi_{\hat{l}_{0}}\right\rangle \|^{2}\right)^{1 / 2} \leqq c n^{-1 / 2}\|a\|(\|\hat{l}\|+c),
$$

where we have used Relation (4.4). This proves Lemma 4.1.

When we combine this lemma with the completeness Relation (4.6), we obtain the following lemma, the first part of which is due to Simon [Si1]:

Lemma 4.2. For any $a \in g_{c}$ and $\hat{l} \in \hat{\Gamma}$

$$
\lim _{n \rightarrow \infty} \frac{1}{d_{n}} \operatorname{trace}_{h_{n}} e^{\pi_{n}\left(\frac{a}{n}\right)}=\int e^{\hat{\imath}(a)} d \mu_{\hat{\Gamma}}(\hat{l}) .
$$

Moreover, there is a constant $c$, depending on $\hat{l}_{0}$ only such that

$$
\left|d_{n}^{-1} \operatorname{trace}_{h_{n}} e^{\pi_{n}\left(\frac{a}{n}\right)}-\int e^{\hat{\imath}(a)} d \mu_{\hat{\Gamma}}(\hat{l})\right| \leqq c n^{-1 / 2}\|a\| e^{c\|a\|} .
$$

The preceding lemmas show how $\pi_{n}\left(\frac{a}{n}\right)$ approaches $\hat{l}(a)$ in the coherent state labelled by $\hat{l}$. The next results, which seem to be new, describe the fluctuations of 
$\pi_{n}\left(\frac{a}{n}\right)$ around $\hat{l}(a)$ in the coherent state labelled by $\hat{l}$. For this purpose, we introduce the quantity

$$
\Phi_{n, l}(a)=-i n^{1 / 2}\left(\pi_{n}\left(\frac{a}{n}\right)-\hat{l}(a)\right)
$$

and the bilinear forms

$$
\langle a, b\rangle_{l}=-\operatorname{trace}_{h_{1}} P_{1}(\hat{l})\left(\pi_{1}(a)-\hat{l}(a)\right)\left(\pi_{1}(b)-\hat{l}(b)\right)
$$

on $g_{c}$ and labelled by $l$.

We have the following covariance property:

$$
\langle\operatorname{Ad} g a, \operatorname{Ad} g b\rangle_{\operatorname{Ad}^{*} g l}=\langle a, b\rangle_{l} .
$$

Choose $\delta=\delta(\Gamma)$ such that

$$
\left\|\pi_{1}(a)-\hat{l}(a)\right\| \leqq \delta\|a\|
$$

for all $\hat{l} \in \hat{\Gamma}$. Thus

$$
\left|\langle a, a\rangle_{l}\right| \leqq \delta^{2}\|a\|^{2}
$$

Also set

$$
\operatorname{trace}_{h_{n}}\left(P_{n}(\hat{l}) \cdot\right)=\left\langle\langle\cdot\rangle_{n, l} .\right.
$$

Lemma 4.3. For any $a \in g_{c}, \hat{l} \in \hat{\Gamma}$

$$
\lim _{n \rightarrow \infty}\left\langle\left\langle\exp i \Phi_{n, l}(a)\right\rangle_{n, l}=\exp -\frac{1}{2}\langle a, a\rangle_{l} .\right.
$$

Moreover

$$
\left|\left\langle\exp i \Phi_{n, l}(a)\right\rangle_{n, l}-\exp -\frac{1}{2}\langle a, a\rangle_{l}\right| \leqq 22 n^{-1 / 2}(\delta\|a\|)^{3} e^{\delta^{2}\|a\|^{2}}
$$

for all $n \geqq 4(\delta\|a\|)^{2}$.

Proof. By Relation (4.1)

$$
\left.\left\langle\left\langle\exp i \Phi_{n, l}(a)\right\rangle\right\rangle_{n, l}=\left(\left\langle\exp i \Phi_{1, l}\left(n^{-1 / 2} a\right)\right\rangle\right\rangle_{1, l}\right)^{n},
$$

so we introduce the quantity

$$
G(s)=\operatorname{trace}_{h_{1}} P_{1}(\hat{l}) e^{s^{-1}\left(\pi_{1}(a)-\hat{\imath}(a)\right)} .
$$

Now

$$
G(s)=1-\frac{1}{2 s^{2}}\langle a, a\rangle_{l}+G^{1}(s)
$$

with

$$
\left|G^{1}(s)\right| \leqq \sum_{k=3}^{\infty}(k !)^{-1}\left(s^{-1} \delta\|a\|\right)^{k} \leqq \frac{1}{6}\left(\frac{\delta\|a\|}{s}\right)^{3} e^{\frac{\delta\|a\|}{s}}
$$


from which the first part of the lemma easily follows. From now on, we will assume

$$
s \geqq \operatorname{Max}(1,2 \delta\|a\|) \text {. }
$$

But then

$$
\begin{gathered}
|G(s)| \leqq 1+\left(\frac{\delta\|a\|}{s}\right)^{2}, \\
|G(s)| \geqq \frac{1}{2} .
\end{gathered}
$$

In particular

$$
\left.|G(s)|\right|^{2} \leqq e^{\delta^{2}\|a\|^{2}} .
$$

To prove (4.22), it is obviously sufficient to show

$$
\left|\frac{d}{d s} G(s)^{s^{2}}\right| \leqq 22 \frac{(\delta\|a\|)^{3}}{s^{2}} e^{\delta^{2}\|a\|^{2}} .
$$

But

$$
\frac{d}{d s}\left(G(s)^{s^{2}}\right)=\left(2 s \ln G(s)+\frac{s^{2}}{G(s)} \frac{d}{d s} G(s)\right)(G(s))^{s^{2}} .
$$

Using (4.26) and the estimate (4.29), we easily obtain

$$
\left|\frac{s^{2}}{G(s)} \frac{d G^{1}(s)}{d s}\right| \leqq 4(\delta\|a\|)^{3} \frac{1}{s^{2}} .
$$

Furthermore, the estimate

$$
\left|(1+z)^{-1}-1\right| \leqq 2|z| \text { for }|z| \leqq \frac{1}{2}
$$

gives

$$
\left|G(s)^{-1}-1\right| \leqq 2\left(\frac{\delta\|a\|}{s}\right)^{2}
$$

Hence

$$
s^{2} G(s)^{-1}\left(-\frac{1}{s^{3}}\langle a, a\rangle_{l}\right)=-\frac{1}{s}\langle a, a\rangle_{l}+G^{2}(s)
$$

with

$$
\left|G^{2}(s)\right| \leqq 2 \frac{(\delta\|a\|)^{3}}{s^{2}}
$$

The estimate

$$
|\ln (1-x+y)+x| \leqq 2\left(|x|^{2}+2|y|\right) \quad \text { for } \quad|x|<\frac{1}{4},|y|<\frac{1}{4}
$$

leads to

$$
\ln G(s)=\frac{-1}{2 s^{2}}\langle a, a\rangle_{l}+G^{3}(s)
$$


with

$$
\left|2 s G^{3}(s)\right| \leqq 16 \frac{(\delta\|a\|)^{3}}{s^{2}} .
$$

Collecting terms yields (4.31), concluding the proof of the lemma.

Remark. We note the close similarity of the first part of Lemma 4.3 and its proof to the content and one of the proofs respectively of the central limit theorem in probability theory (see e.g. [Si2]).

We want to give some further insight into the properties of $\Phi_{n, l}$ for $n$ large. First $\langle,\rangle_{l}$, as given by (4.1) is positive semidefinite on $g$, so let $g_{l}=g / \operatorname{ker}\langle,\rangle_{l}$. We claim that $g_{l}$ may be identified with $T_{l} \Gamma$, the tangent space to $\Gamma$ at $l$. To see this, consider the surjective map $l_{l}: g \rightarrow T_{l} \Gamma$ given as

$$
l_{l}(a)=l \circ a d a \text {. }
$$

We have to show that

$$
\operatorname{ker}\langle,\rangle_{l} \cong \operatorname{ker} l_{l} \text {. }
$$

By (4.17) it suffices to restrict attention to the case $l=l_{0}$. Now if a is in ker $l_{l_{0}}$, then

$$
\left\langle\psi(t)\left|\pi_{1}(b)\right| \psi(t)\right\rangle=\left\langle\psi_{\hat{l}_{0}}\left|\pi_{1}(b)\right| \psi_{\hat{l}_{0}}\right\rangle
$$

for all $t$ with

$$
|\psi(t)\rangle=\exp t \pi_{1}(a)\left|\psi_{\hat{l}_{0}}\right\rangle
$$

as follows by differentiating the left hand side of (4.42) with respect to $t$. But Relation (4.42) is only possible if $|\psi(t)\rangle$ is a multiple exptz of $\left|\psi_{\hat{l}_{0}}\right\rangle$ [Si1], so in particular $\pi_{1}(a)\left|\psi_{\hat{l}_{0}}\right\rangle=z\left|\psi_{\hat{l}_{0}}\right\rangle$. By the definition of $\hat{l}_{0}(a)$, this gives $a \in \operatorname{ker}\langle,\rangle_{l_{0}}$, showing $\operatorname{ker} l_{l_{0}} \subseteq \operatorname{ker}\langle,\rangle_{l_{0}}$.

As for the converse, assume now $a \in \operatorname{ker}\langle,\rangle_{l_{0}}$. By the Schwarz inequality, this means that

$$
\pi_{1}(a)\left|\psi_{\hat{l}_{0}}\right\rangle=\hat{l}_{0}(a)\left|\psi_{\hat{l}_{0}}\right\rangle
$$

In particular, this yields

$$
\left\langle\psi_{\hat{l}_{0}}\left|\left[\pi_{1}(a), \pi_{1}(b)\right]\right| \psi_{l_{0}}\right\rangle=0
$$

for all $b \in g$, showing $\operatorname{ker}\langle,\rangle_{l_{0}} \subseteq \operatorname{ker} l_{l_{0}}$, proving our claim.

By construction $\langle\cdot, \cdot\rangle_{l}$ induces a positive definite scalar product on $g_{l}$, which we continue to denote by the same symbol. Also let $g_{l}^{*}$ be the real dual of $g_{l} \cdot g_{l}^{*}$ is, of course, linearly isomorphic to $T_{l}^{*} \Gamma$, the cotangent space to $\Gamma$ at $l$. Let $\langle,\rangle_{l}$ also denote the scalar product on $g_{l}^{*}$ obtained by a duality transformation from $\langle,\rangle_{l}$ on $g_{l}$. Let $d \tau\left(\tau \in g_{l}^{*}\right)$ denote the Lebesgue measure on $g_{l}^{*}$ and consider the probability measure

$$
d \varrho_{l}(\tau)=N^{-1} \exp -\frac{1}{2}\langle\tau, \tau\rangle_{l} d \tau,
$$

$N$ being the appropriate normalization factor. Now let $a \in g$. Then by our previous discussion $l_{a}=l \circ a d a$ is an element of $g_{l} \cong T_{l} \Gamma$. Furthermore, let $\{a\} \in g_{l}^{*}$ be the 
image of $a \in g$ under the homomorphism given by (2.4). With this notation established, consider the selfadjoint operators $\Phi_{l}(a)$ on $L^{2}\left(g_{l}^{*}, d \varrho_{l}\right)$, indexed by $a \in g$ and defined as

$$
\left(e^{i \Phi_{l}(a)} f\right)(\tau)=e^{-i \tau\left(l_{a}\right)} f\left(\tau+\frac{1}{2}\{a\}\right)
$$

for $f \in L^{2}\left(g_{l}^{*}, d \varrho_{l}\right)$. Since $\{a\}\left(l_{a^{\prime}}\right)=-l\left(\left[a, a^{\prime}\right]\right)$, we have the Heisenberg commutation relations

$$
e^{i \Phi_{l}(a)} e^{i \Phi_{l}\left(a^{\prime}\right)}=e^{i \Phi_{l}\left(a+a^{\prime}\right)+\frac{i}{2} l\left[\left(a, a^{\prime}\right]\right)},
$$

or in infinitesimal form

$$
\left[\Phi_{l}(a), \Phi_{l}\left(a^{\prime}\right)\right]=-i l\left(\left[a, a^{\prime}\right]\right) .
$$

Of course, we also have

$$
\Phi_{l}\left(a+a^{\prime}\right)=\Phi_{l}(a)+\Phi_{l}\left(a^{\prime}\right), \quad \Phi_{l}(t a)=t \Phi_{l}(a) .
$$

Because of these relations, we view these operators as quantizing the symplectic structure discussed in Sect. 1 . Let furthermore $\langle\cdot\rangle_{l}$ denote the expectation of an operator in $L^{2}\left(g_{l}^{*}, d \varrho_{l}\right)$ with respect to the state given by the wavefunction, which is identically equal to one on $g_{l}^{*}$.

Then by (4.44)

$$
\left\langle e^{i \Phi_{l}(a)}\right\rangle_{l}=N^{-1} \int e^{-i \tau\left(l_{a}\right)} e^{-\frac{1}{2}\langle\tau, \tau\rangle} d \tau .
$$

To evaluate this integral, let $l_{a}^{\#} \in g_{l}^{*}$ be such that $\tau\left(l_{a}\right)=\left\langle\tau, l_{a}^{\#}\right\rangle_{l}$ and therefore $\left\langle l_{a}^{\#}, l_{a}^{\#}\right\rangle_{l}=\left\langle l_{a}, l_{a}\right\rangle_{l}$. Then, by standard calculations on Gaussian integrals, we obtain

$$
\left.\left\langle e^{i \Phi_{l}(a)}\right\rangle\right\rangle_{l}=e^{-\frac{1}{2}\left\langle l_{a}^{\#}, l_{a}^{\sharp \ddagger}\right\rangle_{l}}=e^{-\frac{1}{2}\langle a, a\rangle_{l}} .
$$

Therefore, the first part of Lemma 4.3 takes the more suggestive form

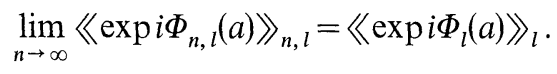

The relationship between $\Phi_{n, l}$ and $\Phi_{l}$ goes even further. Note that

$$
\left[\Phi_{n, l}(a), \Phi_{n, l}\left(a^{\prime}\right)\right]=-\Pi_{n}\left(\frac{1}{n}\left[a, a^{\prime}\right]\right),
$$

which formally tends to (4.46) as $n \rightarrow \infty$. In fact, by extending $\Phi_{l}(a)$ to $a \in g_{c}$ in the obvious way, we have

Lemma 4.4. For any $a_{1}, \ldots, a_{k} \in g_{c}$,

$$
\lim _{n \rightarrow \infty}\left\langle\left\langle\prod_{\kappa=1}^{k} e^{i \Phi_{n, l}\left(a_{\kappa}\right)}\right\rangle_{n, l}=\left\langle\left\langle\prod_{\kappa=1}^{k} e^{i \Phi_{l}\left(a_{\kappa}\right)}\right\rangle_{l} .\right.\right.
$$

In fact, there is $\delta_{1}=\delta_{1}(\Gamma)$ such that

$$
\left|\left\langle\left\langle\prod_{\kappa=1}^{k} e^{i \Phi_{n, l}\left(a_{\kappa}\right)}\right\rangle\right\rangle_{n, l}-\left\langle\left\langle\prod_{\kappa=1}^{k} e^{i \Phi_{l}\left(a_{\kappa}\right)}\right\rangle\right\rangle\right| \leqq 14 n^{-\frac{1}{8}} e^{\delta_{1}^{2}\left(\sum_{\kappa=1}^{k}\left\|a_{\kappa}\right\|\right)^{2}} .
$$

provided $n \geqq\left(\delta_{1} \sum_{\kappa=1}^{k}\left\|a_{\kappa}\right\|\right)^{8}$. 
Proof. We want to rewrite the expressions involved such that Lemma 4.3 may be applied. Now the right hand side of Relation (4.52) equals

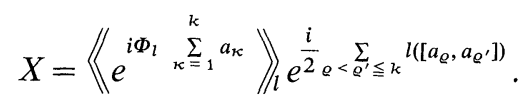

To rewrite the left hand side in a comparable form, we will use the BakerCampbell-Hausdorff formula as follows: Given the group $G$, there is $0<\varepsilon_{0}<1, \alpha>1$ and a map $b: g_{c} \times g_{c} \rightarrow g_{c}$ with the following properties: For all $a, a^{\prime}$ with $\|a\|+\left\|a^{\prime}\right\|<\varepsilon_{0}$

$$
\exp a \exp a^{\prime}=\exp \left(a+a^{\prime}+\frac{1}{2}\left[a, a^{\prime}\right]+b\left(a, a^{\prime}\right)\right)
$$

with

$$
\left\|b\left(a, a^{\prime}\right)\right\| \leqq \alpha^{2}\|a\|\left\|a^{\prime}\right\|\left(\|a\|+\left\|a^{\prime}\right\|\right) .
$$

By modifying $\alpha$ if necessary, we may also assume that

$$
\left\|\frac{1}{2}\left[a, a^{\prime}\right]\right\| \leqq \alpha\|a\|\left\|a^{\prime}\right\|
$$

for all $a, a^{\prime} \in g_{c^{*}}$. Next, define $a^{\kappa}$ and $b^{\kappa}(1 \leqq \kappa \leqq k)$ inductively as $a^{1}=a_{1}, b^{1}=0$ and

$$
\begin{gathered}
\exp n^{-1 / 2} a^{\kappa}=\exp n^{-1 / 2} a^{\kappa-1} \exp n^{-1 / 2} a_{\kappa}, \\
b^{\kappa}=b^{\kappa-1}+n^{1 / 2} b\left(n^{-1 / 2}\left(a^{\kappa-1}+b^{\kappa-1}\right), n^{-1 / 2} a_{\kappa}\right) \\
+\frac{n^{-1 / 2}}{2}\left[b^{\kappa-1}, a_{\kappa}\right]+\frac{n^{-1}}{4} \sum_{\varrho<\varrho^{\prime} \leqq \kappa-1}\left[\left[a_{\varrho}, a_{\varrho^{\prime}}\right], a_{\kappa}\right],
\end{gathered}
$$

such that we have the alternative relation

$$
b^{\kappa}=a^{\kappa}-\sum_{\varrho \leqq \kappa} a_{\varrho}-\frac{n^{-1 / 2}}{2} \sum_{\varrho<\varrho^{\prime} \leqq k}\left[a_{\varrho}, a_{\varrho^{\prime}}\right] .
$$

Note that this definition makes sense for all large $n$ and fixed $a_{1}, \ldots, a_{k}$. We want to estimate $a^{k}$ and $b^{k}$, so we proceed by induction:

$$
\begin{aligned}
\left\|a^{\kappa}\right\| \leqq & \left\|a^{\kappa-1}\right\|+\left\|a_{\kappa}\right\|+n^{-1 / 2} \alpha\left\|a^{\kappa-1}\right\|\left\|a_{\kappa}\right\| \\
& +n^{-1} \alpha^{2}\left\|a^{\kappa-1}\right\|\left\|a_{\kappa}\right\|\left(\left\|a^{\kappa-1}\right\|+\left\|a_{\kappa}\right\|\right), \\
\left\|b^{\kappa}\right\| \leqq & \left\|b^{\kappa-1}\right\|+n^{-1} \alpha^{2}\left(\left\|a^{\kappa-1}\right\|+\left\|b^{\kappa-1}\right\|\right)\left\|a_{\kappa}\right\| \\
& \cdot\left(\left\|a^{\kappa-1}\right\|+\left\|a_{\kappa}\right\|+\left\|b^{\kappa-1}\right\|\right) \\
& +n^{-1 / 2} \alpha\left\|b^{\kappa-1}\right\|\left\|a_{\kappa}\right\|+n^{-1} \alpha^{2}\left(\sum_{\varrho \leqq \kappa-1}\left\|a_{\varrho}\right\|\right)^{2}\left\|a_{\kappa}\right\| .
\end{aligned}
$$

Now let $n$ be so large that

$$
4 n^{-1 / 2} \alpha \sum_{\varrho \leqq k}\left\|a_{\varrho}\right\| \leqq \varepsilon_{0}<1 .
$$

Then an easy induction argument shows that

$$
\left\|a^{\kappa}\right\| \leqq\left\|a_{1}\right\|+2 \sum_{\varrho \leqq \kappa}\left\|a_{\varrho}\right\|
$$


and

$$
\left\|b^{\kappa}\right\| \leqq n^{-3 / 4} \sum_{\varrho \leqq \kappa}\left\|a_{\varrho}\right\|
$$

Now we write

Let

$$
\begin{aligned}
& Y=\left\langle\left\langle\prod_{\kappa=1}^{k} \operatorname{expi\Phi _{n,l}(a_{\kappa })}\right\rangle_{n, l}\right. \\
& =\left\langle\left\langle\exp i \Phi_{n, l}\left(a^{k}\right)\right\rangle\right\rangle_{n, l} \exp \left[\operatorname{in}^{1 / 2} l\left(b^{k}\right)+\frac{i}{2} l\left(\sum_{\varrho<\varrho^{\prime} \leqq k}\left[a_{\varrho}, a_{\varrho^{\prime}}\right]\right)\right] \text {. }
\end{aligned}
$$

$$
Z=e^{-\frac{1}{2}\left\langle a^{k}, a^{k}\right\rangle_{l}} \exp \left[i n^{1 / 2} l\left(b^{k}\right)+\frac{i}{2} l\left(\sum_{\varrho<\varrho^{\prime} \leqq k}\left[a_{\varrho}, a_{\varrho^{\prime}}\right]\right)\right] .
$$

Then by Lemma 4.3, (4.62), and (4.64)

$$
|Z-Y| \leqq 6 n^{-1 / 8} \exp \left[\left(\delta^{\prime} \sum_{\varrho=1}^{k}\left\|a_{\varrho}\right\|\right)^{2}\right]
$$

if we choose $\alpha$ so large that $\alpha \geqq\|l\|$ and $\left(\delta^{\prime}\right)^{2} \geqq 4 \delta^{2}+\alpha\|l\|$ for all $l \in \Gamma$. Again, Relations (4.62) and (4.64) give

$$
\left|e^{i n^{1 / 2} l\left(b^{k}\right)}-1\right| \leqq 4 n^{-1 / 4} \sum_{\varrho=1}^{k}\left\|a_{\varrho}\right\| \leqq n^{-1 / 8} .
$$

Also by (4.60), (4.62), (4.63), and (4.64)

$$
\left|\left\langle a^{k}, a^{k}\right\rangle_{l}-\left\langle\sum_{\varrho=1}^{k} a_{\varrho}, \sum_{\varrho=1}^{k} a_{\varrho}\right\rangle_{l}\right| \leqq 3\left(\sum_{\varrho=1}^{k}\left\|a_{\varrho}\right\|\right)\left(n^{-1 / 2} \alpha\left(\sum_{\varrho=1}^{k}\left\|a_{\varrho}\right\|\right)^{2}+\left\|b^{k}\right\|\right) \leqq n^{-1 / 8} \text {. }
$$

Therefore, we obtain

$$
|X-Z| \leqq 8 n^{-1 / 8} \exp \left[\left(\delta^{\prime} \sum_{\varrho=1}^{k}\left\|a_{\varrho}\right\|\right)^{2}\right] .
$$

Combining (4.67) and (4.70) proves the lemma once we choose

$$
\delta_{1}=\operatorname{Max}\left(\delta^{\prime}, \frac{\alpha}{\varepsilon_{0}}\right) \text {. }
$$

In an analogous fashion, we obtain uniform bounds in the following form

Lemma 4.5. There is $\delta_{2}=\delta_{2}(\Gamma)$ such that for any $a_{1}, \ldots, a_{k} \in g_{c}$

$$
\left|\left\langle\left\langle\prod_{\kappa=1}^{k} \exp i \Phi_{n, l}\left(a_{\kappa}\right)\right\rangle\right\rangle_{n, l}\right| \leqq \exp \delta_{2}^{2}\left(\sum_{\kappa=1}^{k}\left\|a_{\kappa}\right\|\right)^{2},
$$

provided

$$
n \geqq \delta_{2}^{2}\left(\sum_{\kappa=1}^{k}\left\|a_{\kappa}\right\|\right)^{2}
$$


Proof. We slightly modify the arguments of the proof of Lemma 4.4. Replace condition (4.62) by the condition

$$
4 n^{-1 / 2} \alpha \sum_{\varrho=1}^{k}\left\|a_{\varrho}\right\| \leqq \varepsilon_{0}<1 .
$$

Then (4.63) still holds, proving the lemma with $\delta_{2}^{2} \geqq \operatorname{Max}\left(\delta_{1}^{2}, \frac{4 \alpha}{\varepsilon_{0}}\right)$. The next two lemmas allow us to prove convergence rates and bounds of the moments in a form we will need in Sect. 6.

Lemma 4.6. There is $C_{1}=C_{1}(\Gamma)$ such that for all $a_{0}, a_{1}, \ldots, a_{k} \in g_{c}$

$$
\begin{aligned}
\left|\left\langle\prod_{\kappa=1}^{k_{1}} \Phi_{n, l}\left(a_{\kappa}\right) \exp i \Phi_{n, l}\left(a_{0}\right) \prod_{\kappa=k_{1}+1}^{k} \Phi_{n, l}\left(a_{\kappa}\right)\right\rangle\right\rangle_{n, l} \\
\quad-\left\langle\left\langle\prod_{\kappa=1}^{k_{1}} \Phi_{l}\left(a_{\kappa}\right) \exp i \Phi_{l}\left(a_{0}\right) \prod_{\kappa=k_{1}+1}^{k} \Phi_{l}\left(a_{\kappa}\right)\right\rangle\right\rangle_{l} \mid \\
\leqq n^{-1 / 8} C_{1}\left(\prod_{\kappa=1}^{k}\left\|a_{\kappa}\right\|\right) k^{k} e^{C_{1}\left\|a_{0}\right\|^{2}},
\end{aligned}
$$

provided $n \geqq\left(C_{1}\left(1+\left\|a_{0}\right\|\right)^{2}\right)^{2}$.

Proof. The proof follows from an application of Cauchy's theorem. Indeed, consider

$$
\begin{aligned}
& S^{n}\left(z_{1}, \ldots, z_{k}\right)-S\left(z_{1}, \ldots, z_{k}\right) \\
& =\left\langle\left\langle\prod_{\kappa=1}^{k_{1}} \exp i \Phi_{n, l}\left(z_{\kappa} a_{\kappa}\right) \exp i \Phi_{n, l}\left(a_{0}\right) \prod_{\kappa=k_{1}+1}^{k} \exp i \Phi_{n, l}\left(z_{\kappa} a_{\kappa}\right)\right)\right\rangle_{n, l} \\
& \quad-\left\langle\left\langle\prod_{\kappa=1}^{k_{1}} \exp i \Phi_{l}\left(z_{\kappa} a_{\kappa}\right) \exp i \Phi_{l}\left(a_{0}\right) \prod_{\kappa=k_{1}+1}^{k} \exp i \Phi_{l}\left(z_{\kappa} a_{\kappa}\right)\right\rangle\right\rangle_{l} .
\end{aligned}
$$

Then, the left hand side of $(4.75)$ is the absolute value of

$$
\left.\prod_{\kappa=1}^{k}\left(-i \frac{d}{d z_{\kappa}}\right)\left(S^{n}-S\right)\left(z_{1}, \ldots, z_{k}\right)\right|_{z_{\kappa}=0},
$$

and hence bounded by

$$
\left(\prod_{\kappa=1}^{k} \frac{1}{r_{\kappa}}\right) \sup _{\left|z_{\kappa}\right|=r_{\kappa}}\left|\left(S^{n}-S\right)\left(z_{1}, \ldots, z_{k}\right)\right| .
$$

Since we may assume $a_{\kappa} \neq 0$, we take $r_{\kappa}=\left(k\left\|a_{\kappa}\right\|\right)^{-1}$. Hence, estimate (4.75) follows from Lemma 4.4. q.e.d.

The expectations of monomials of order $k$ in the $\Phi_{l}$ with respect to $\langle\cdot\rangle_{l}$ show the typical $k^{k / 2}$ behaviour of operators satisfying Heisenberg group commutation relations or of Gaussian random variables. In order to obtain the convergence factor $n^{-1 / 8}$ in (4.75), we had to pay a price, namely a $k^{k}$ behaviour only. However, the next lemma shows that we may obtain bounds on expectations of monomials of the $\Phi_{n, l}$ of the desired form which are uniform in $n$. 
Lemma 4.7. There is $C_{2}=C_{2}(\Gamma)$ such that uniformly for all $n$, all $a_{1}, \ldots, a_{k} \in g_{c}$ and $a_{0} \in g$

$$
\begin{aligned}
& \left|\left\langle\left\langle\prod_{\kappa=1}^{k_{1}} \Phi_{n, l}\left(a_{\kappa}\right) \exp i \Phi_{n, l}\left(a_{0}\right) \prod_{\kappa=k_{1}+1}^{k} \Phi_{n, l}\left(a_{\kappa}\right)\right\rangle\right\rangle_{n, l}\right| \\
& \leqq\left(C_{2}\left(1+\left\|a_{0}\right\|^{2}\right) k\right)^{k / 2} e^{C_{2}\left\|a_{0}\right\|^{2}} \cdot \prod_{\kappa=1}^{k}\left\|a_{\kappa}\right\| .
\end{aligned}
$$

Proof. As in the proof of Lemma 4.6, we bound the left hand side of (4.79) by

$$
\left(\prod_{\kappa=1}^{k} r_{\kappa}^{-1}\right) \sup _{\left|z_{\kappa}\right|=r_{\kappa}}\left|S^{n}\left(z_{1}, \ldots, z_{n}\right)\right| \text {. }
$$

Now choose $r_{\kappa}=\left(\sqrt{k}\left\|a_{\kappa}\right\|\right)^{-1}$. By Lemma 4.5, the bound (4.79) now follows for all $n \geqq \delta_{2}^{2}\left(\sqrt{k}+\left\|a_{0}\right\|\right)^{2}$. On the other hand, since $\exp i \Phi_{n, l}\left(a_{0}\right)$ is unitary for $a_{0} \in g$ and $\left\|\Phi_{n, l}\left(a_{\kappa}\right)\right\| \leqq C^{\prime}\left\|a_{\kappa}\right\| n^{1 / 2}\left(C^{\prime}=C^{\prime}(\Gamma)\right)$, for all $n \leqq \delta_{2}^{2}\left(\sqrt{k}+\left\|a_{0}\right\|\right)^{2}$, the left hand side of (4.79) is bounded by

$$
\prod_{\kappa=1}^{k}\left(C^{\prime}\left\|a_{\kappa}\right\| n^{1 / 2}\right) \leqq\left(C^{\prime} \delta_{2}^{2}\left(1+\left\|a_{0}\right\|\right)^{2} k\right)^{k / 2} .
$$

This proves Lemma 4.7 .

We conclude this section with a trivial remark which, however, will be useful for our discussion of the quantum fluctuations around classical orbits, to be given in Sect. 6. We note that $\Phi_{l}(a)$ and $\Phi_{\mathrm{Ad}^{*} g l}(\operatorname{Ad} g a)$ satisfy the same commutation relations and have the same expectations. In fact, it is not difficult to construct a unitary equivalence given by a unitary operator $\mathrm{Ad}^{\sim} \mathrm{g}$

$$
\operatorname{Ad}^{\sim} g: L^{2}\left(g_{l}^{*}, d \varrho_{l}\right) \rightarrow L^{2}\left(g_{\mathrm{Ad}^{*} g l}^{*}, d \varrho_{\mathrm{Ad}^{*} g l}\right),
$$

such that

$$
\operatorname{Ad}^{\sim} g \Phi_{l}(a)\left(\mathrm{Ad}^{\sim} g\right)^{-1}=\Phi_{\mathrm{Ad}^{*} g l}(\operatorname{Ad} g a)
$$

\section{Classical Limits for Partition Functions}

We define the classical partition function to be

$$
Z_{\beta, \Gamma}^{c l}=(2 \pi)^{-v} \int_{\mathbb{R}^{2} \times \Gamma} d^{v} x d^{v} p d \mu_{\Gamma}(l) \exp -\beta\left(\frac{p^{2}}{2 m}+V(x)+l\left(A_{0}(x)\right) .\right.
$$

We recall that by our assumptions the Yang-Mills potentials are $C_{0}^{\infty}$. Also we assume the condition (3.3) on the scalar potential $V$ to be satisfied. Therefore these assumptions imply that $Z_{\beta, \Gamma}^{c l}$ is finite. The aim of this section is to prove

Theorem 5.1. With the above assumptions, the scaled quantum statistical partition function converges to the classical partition function

$$
\lim _{\substack{h \rightarrow 0 \\ n \hbar=1}} \hbar^{v} Z_{\beta, \pi_{n}, \hbar}=Z_{\beta, \Gamma}^{c l} .
$$


Proof. By performing the $p$-integration in the defining relation (5.1), it easily suffices to show

$$
\begin{aligned}
& \lim _{\substack{t \rightarrow 0 \\
n=\left(\beta(m t)^{-1}\right)^{1 / 2}}} t^{v / 2} d_{n}^{-1} \operatorname{Trace}_{\mathscr{H}_{\pi_{n}}} e^{-t H(t)}=(2 \pi)^{-v / 2} \int_{\mathbb{R}^{\nu \times \Gamma}} d^{v} x d \mu_{\Gamma}(l) \exp -\beta\left(V(x)+l\left(A_{0}(x)\right)\right) \\
& \text { where } t=\frac{\beta \hbar^{2}}{m} \text { and }
\end{aligned}
$$

$$
H(t)=-\frac{1}{2} \sum_{j=1}^{v}\left(\partial_{j}+\pi_{n}\left(A_{j}\right)\right)^{2}+\pi_{n}\left(\frac{i \beta}{n t} A_{0}\right)+\frac{\beta}{t} V .
$$

We now give a representation of the kernel of exp $-t H(t)$ in terms of the Brownian motion. Let $\omega$ be a Brownian path from $x$ to $y$ in time $t$. To simplify the presentation, we now assume that $G$ (and its complexification $G_{c}$ ) is a matrix group. Let $g_{t}(\omega) \in G_{c}$ be the solution of Ito's stochastic differential equation [Po]

$$
\begin{aligned}
g_{t^{\prime}}(\omega)= & 1+\int_{0}^{t^{\prime}}\left\{\sum_{v=1}^{v} d \omega_{j}(s) A_{j}(\omega(s))+d s\left[\frac { 1 } { 2 } \sum _ { j = 1 } ^ { v } \left[\partial_{j} A_{j}(\omega(s))+A_{j}\left((\omega(s))^{2}\right]\right.\right.\right. \\
& \left.\left.-\frac{i \beta}{t n} A_{0}(\omega(s))\right]\right\} g_{s}(\omega) ; \quad 0 \leqq t^{\prime} \leqq t .
\end{aligned}
$$

Then the kernel of exp $-t H(t)$ may be represented by

$$
\int d P_{x, y}^{t}(\omega) \exp \left[-\frac{\beta}{t} \int_{0}^{t} V(\omega(s)) d s\right] \pi_{n}\left(g_{t}(\omega)\right),
$$

where $d P_{x, y}^{t}(\omega)$ is the conditional Wiener measure. In order to estimate $\pi_{n}\left(g_{t}(\omega)\right)$ for the interesting case $x=y$, we rewrite $g_{t}(\omega)$ with the help of the Brownian bridge $\alpha(\cdot)$ (see e.g. [Si2]).

$$
\begin{aligned}
g_{t}= & +\int_{0}^{t^{\prime} / t}\left\{\sqrt{t} \sum_{j=1}^{v} d \alpha_{j}(s) A_{j}(x+\sqrt{t} \alpha(s))\right. \\
& +t d s \frac{1}{2} \sum_{j=1}^{v}\left[\partial_{j} A_{j}(x+\sqrt{t} \alpha(s))+A_{j}(x+\sqrt{t} \alpha(s))^{2}\right] \\
& \left.-i \frac{\beta}{n} d s \cdot A_{0}(x+\sqrt{t} \alpha(s))\right\} g_{s t} .
\end{aligned}
$$

If we iterate this once and use the abbreviations

$$
\begin{aligned}
a_{j, s} & =\sqrt{\beta m} A_{j}(x+\sqrt{t} \alpha(s)), \\
a_{0, s} & =\beta A_{0}(x+\sqrt{t} \alpha(s)), \\
v_{s} & =\beta V(x+\sqrt{t} \alpha(s)),
\end{aligned}
$$

and the relation $t=n^{-2} \beta m^{-1}$, we obtain

$$
g_{t}=\exp \left\{\left[\int_{0}^{1} \frac{1}{n}\left(\sum_{j=1}^{v} d \alpha_{j}(s) a_{j, s}\right)-\frac{i}{n} d s a_{0, s}\right]+O\left(n^{-2}\right)\right\}
$$


where $O\left(n^{-2}\right)$ denotes an element in $g_{c}$ of norm $O\left(n^{-2}\right)$ uniformly in $\alpha$. By (4.2) this gives

$$
\pi_{n}\left(g_{t}\right)=\exp \left\{\left[\int_{0}^{1} \frac{1}{n} \sum_{j=1}^{v} d \alpha_{j} \pi_{n}\left(a_{j, s}\right)-\frac{i}{n} d s \pi_{n}\left(a_{0, s}\right)\right]+O\left(\frac{1}{n}\right)\right\},
$$

where $O\left(\frac{1}{n}\right)$ now denotes an endomorphism of $h_{n}$ of norm $O\left(\frac{1}{n}\right)$ uniformly in $\alpha$.

Let $E_{\alpha}(\cdot)$ be the expectation with respect to the Brownian bridge. Thus

$$
\int \cdot d P_{x, y}^{t}=(2 \pi t)^{-v / 2} e^{-\frac{|x-y|^{2}}{2 t}} E_{\alpha}(\cdot) .
$$

In the appendix we will give a proof of the following lemma

Lemma 5.2. For any $\varepsilon>0$ there is $n(\varepsilon)$ such that for all $n \geqq n(\varepsilon)$

$$
\begin{aligned}
& \frac{1}{d_{n}} \mid \int d^{v} x \operatorname{trace}_{h_{n}}\left\{E_{\alpha}\left(\exp \left[-\int_{0}^{1}\left(i \pi_{n}\left(\frac{1}{n} a_{0, s}\right)+v_{s}\right) d s+\sum_{j=1}^{v} \int_{0}^{1} \pi_{n}\left(\frac{1}{n} a_{j, s}\right) d \alpha_{j}(s)\right]\right)\right. \\
& \left.-e^{-\beta V(x)-i \beta \pi_{n}\left(\frac{1}{n} A_{0}(x)\right)}\right\} \mid<\varepsilon \text {. }
\end{aligned}
$$

Therefore, to prove Theorem 5.1, it suffices to show

$$
\lim _{n \rightarrow \infty} \frac{1}{d_{n}} \int d^{v} x e^{-\beta V(x)} \operatorname{trace}_{h_{n}} e^{-i \beta \pi_{n}\left(\frac{1}{n} A_{0}(x)\right)}=\int d^{v} x d \mu_{\Gamma}(l) e^{-\beta\left(V(x)+l\left(A_{0}(x)\right)\right.} .
$$

But this follows easily from Lemma 4.2 and the Lebesgue bounded convergence theorem.

We note that Theorem 5.1 may also be extended to the case which includes an external metric field. The way is to combine the methods used here with those applied in [Ho], which control the metric field. An entirely different proof which covers all such external fields simultaneously will be given in [ST].

\section{The Classical Limit for the Equations of Motion}

In this section we extend Hepp's discussion $[\mathrm{H}]$ of the Ehrenfest solutions to the motion in external Yang-Mills potentials. In particular we will determine the quantum fluctuations around the classical solution. We incidentally remark, that it is possible to include external metric fields simultaneously, the case of external metric fields alone having been discussed in [Ho]. To simplify the proofs we assume in this section that $V$ (together with the $A_{\mu}$ ) is $C_{0}^{\infty}$, although extensions to more general $V$ (and $A_{u}$ ) are possible.

Define Weyl operators

$$
U(\alpha)=\exp i\left(\alpha \cdot b-\alpha^{*} \cdot b^{*}\right)
$$

with $b^{*}, b$ being the usual creation and annihilation operators for the harmonic oscillator

$$
b_{j}=\left(q_{j}+i p_{j}\right) 2^{-1 / 2}
$$


and

$$
\alpha_{j}=\left(\xi_{j}+i \pi_{j}\right) 2^{-1 / 2}
$$

for $(\xi, \pi) \in \mathbb{R}^{2 v}$. Also $q_{j}$ is the multiplication operator by $x_{j}$ and $p_{j}=-i \partial_{j}$. We have

$$
U(\alpha) b U(\alpha)^{*}=b-\alpha .
$$

Also we set

$$
q_{\hbar}=\hbar^{1 / 2} q, \quad p_{\hbar}=\hbar^{1 / 2} p
$$

and (see Sect. 3 for the definition of $H_{\hbar}^{2}$ )

$$
U_{\hbar}(t)=\exp -\frac{i}{\hbar} H_{\hbar}^{2} t
$$

by which $\hbar$ is introduced in a symmetrical way. Again we fix $\Gamma$ to be the coadjoint orbit $-i \hat{\Gamma}$, where $\hat{\Gamma}$ is the coadjoint orbit in $g_{c}$ through the fundamental weight $\hat{l}_{0}=i l_{0}$. We recall from Sect. 2 that $\left(\xi^{t}, \pi^{t}, \gamma^{t}\right)$ and $\left(\xi^{t}, \pi^{t}, \lambda^{t}\right)$ for $|t|<T$ are solutions of the Wong equations with initial conditions $(\xi, \pi, e)$ and $(\xi, \pi, \lambda)$ respectively such that $\mathrm{Ad}^{*} \gamma^{t} \lambda=\lambda^{t}$. We set $\hat{\lambda}=i \lambda, \hat{\lambda}^{t}=i \lambda^{t}$ and $\alpha^{t}=\left(\xi^{t}+i \pi^{t}\right) 2^{-1 / 2}$. To obtain the ansatz for the Hamiltonian describing the quantum fluctuations, as in $[\mathrm{H}]$ we will expand the Hamiltonian around the classical orbit. The quadratic part will give us the desired operator.

More precisely consider

$$
\begin{aligned}
H_{\hbar}^{2}(t)= & U\left(\hbar^{-1 / 2} \alpha^{t}\right)^{*} \pi_{n}\left(\gamma^{t}\right)^{*} H_{\hbar}^{2} \pi_{n}\left(\gamma^{t}\right) U\left(\hbar^{-1 / 2} \alpha^{t}\right) \\
= & \frac{1}{2 m}\left(\hbar^{1 / 2} p_{j}+\pi_{j}^{t}-i \hbar \pi_{n}\left(\left(\operatorname{Ad} \gamma^{t}\right)^{-1} A_{j}\left(\hbar^{1 / 2} q+\xi^{t}\right)\right)^{2}\right. \\
& +V\left(\hbar^{1 / 2} q+\xi^{t}\right)-i \hbar \pi_{n}\left(\left(\operatorname{Ad} \gamma^{t}\right)^{-1} A_{0}\left(\hbar^{1 / 2} q+\xi^{t}\right)\right) .
\end{aligned}
$$

In order to expand this around $\left(\xi^{t}, \pi^{t}, \lambda^{t}\right)$, we recall the definitions of the quantities $\Phi_{n, \lambda}(n \hbar=1)$

$$
\Phi_{n, \lambda}(a)=\hbar^{-1 / 2}\left(-i \hbar \pi_{n}(a)-\lambda(a)\right)
$$

for $a \in g_{c}$. Using the Wong equations for $\left(\xi^{t}, \pi^{t}, \gamma^{t}\right)$ we obtain

$$
\begin{aligned}
H_{\hbar}^{2}= & H_{c l}\left(\xi^{t}, \pi^{t}, \lambda^{t}\right)+\hbar^{1 / 2}\left(\frac{d \xi_{j}^{t}}{d t} p_{j}-\frac{d}{d t} \pi_{j}^{t} \cdot q_{j}\right) \\
& +\hbar^{1 / 2} \Phi_{n, \lambda}\left(\left(\operatorname{Ad} \gamma^{t}\right)^{-1} \gamma^{t} \frac{d}{d s}\left(\gamma^{t}\right)^{-1}\right)+\hbar H_{f l}^{n}(t)+\hbar^{3 / 2} B^{t} .
\end{aligned}
$$

The quadratic term $H_{f l}^{n}(t)$, which will describe the quantum fluctuations, is given as

$$
\begin{aligned}
H_{f l}^{n}(t)= & \frac{1}{2 m}\left(p_{j}+\lambda^{t}\left(\partial_{k} A_{j}\left(\xi^{t}\right)\right) q^{k}+\Phi_{n, \lambda}\left(\left(\operatorname{Ad} \gamma^{t}\right)^{-1} A_{j}\left(\xi^{t}\right)\right)\right)^{2} \\
& +\frac{1}{m}\left(\pi_{j}^{t}+\lambda^{t}\left(A_{j}\left(\xi^{t}\right)\right)\left(\lambda^{t}\left(\partial_{k} \partial_{l} A_{j}\left(\xi^{t}\right)\right) q_{k} q_{l}+\Phi_{n, \lambda}\left(\left(\operatorname{Ad} \gamma^{t}\right)^{-1} \partial_{k} A_{j}\left(\xi^{t}\right)\right) q_{k}\right)\right. \\
& +\Phi_{n, \lambda}\left(\left(\operatorname{Ad} \gamma^{t}\right)^{-1} \partial_{k} A_{0}\left(\xi^{t}\right)\right) q_{k}+\frac{1}{2} \lambda^{t}\left(\partial_{k} \partial_{l} A_{0}\left(\xi^{t}\right)\right) q_{k} q_{l}+\frac{1}{2} \partial_{k} \partial_{l} V\left(\xi^{t}\right) q_{k} q_{l}
\end{aligned}
$$


$H_{f l}^{n}(t)$ has the following structure: It is quadratic in $q, p$, and $\Phi_{n, \lambda}$ (a "harmonic" oscillator) with coefficients (including Lie algebra elements) which depend on $A_{\mu}$ and $V$ and its derivatives evaluated at $\left(\xi^{t}, \pi^{t}, \lambda^{t}\right)$. In particular these coefficients are bounded for $|t|<T$. Finally $B^{t}$ is of the form

$$
B^{t}=\sum_{1 \leqq i, j, k \leqq 2 v+\operatorname{dim} G} a_{i j k}(t, q, \hbar) X_{i}(n) X_{j}(n) X_{k}(n) .
$$

Here $\left\{X_{i}(n)\right\}_{1 \leqq i \leqq 2 v+\operatorname{dim} G}$ is the family of operators $q_{i}, p_{j}$, and $\Phi_{n, \lambda}\left(e_{m}\right)$ enumerated in an arbitrary way $\left(e_{m}\right.$ a fixed basis in $\left.g\right)$. The $a_{i j k}(t, q, \hbar)$ are $C^{\infty}$ functions in $t(|t|<T)$ and $q$, uniformly bounded in $\hbar$.

To state the main result, let $\Phi_{\lambda}(\cdot)$ be the operators as defined in Sect. 4. Then $H_{f l}(t)$ obtained by substituting $\Phi_{\lambda}$ for $\Phi_{n, \lambda}$ in Relation (6.10) is of a harmonic oscillator type and thus is easily seen to have a dense set of analytic vectors in the Hilbert space $\mathscr{L}_{\lambda}=L^{2}\left(\mathbb{R}^{v}, d^{v} s\right) \otimes L^{2}\left(g_{\lambda}^{*}, d \varrho_{\lambda}\right)$. Therefore this operator defines a selfadjoint operator also denoted by $H_{f l}(t)$. Let $q_{i}(t), p_{i}(t)$, and $\Phi_{\lambda}(t)(a)$ be the unique solutions in the Heisenberg picture in $\mathscr{L}_{\lambda}$ to the operator differential equations

$$
\frac{d}{d t} f(t)=-i\left[f(t), H_{f l}(t)\right]
$$

with initial conditions $q_{i}, p_{i}$, and $\Phi_{\lambda}(a)$ respectively. $q_{i}(t), p_{i}(t)$, and $\Phi_{\lambda}(t)(a)$ may alternatively be given as follows. Consider the unitary operators:

$$
\hat{W}(t, s)=T \exp -i \int_{s}^{t} H_{f l}(u) d u,
$$

where $T$ denotes time ordering. As in $[\mathrm{H}]$ the existence of the $\hat{W}(t, s)$ and their differentiability properties may easily be established using the Dyson series on a dense set. Then

$$
f(t)=\hat{W}(t, 0)^{*} f \hat{W}(t, 0)
$$

is indeed a solution of the Heisenberg equation (6.12) with initial condition $f$, as follows directly by differentiation.

We want to make an additional comment on Eq. (6.12). One might be tempted to introduce the operator $\tilde{H}_{f l}(t)$ on $\mathscr{L}_{\lambda^{t}}$ obtained by substituting $\Phi_{\lambda^{t}}(\cdot)$ for $\Phi_{n, \lambda}\left(\left(\operatorname{Ad} \gamma^{t}\right)^{-1} \cdot\right)$ in (6.10). $\tilde{H}_{f l}(t)$ on $\mathscr{L}_{\lambda t}$ and $H_{f l}(t)$ on $\mathscr{L}_{\lambda}$ are of course unitarity equivalent, as follows from the concluding remarks of Sect. 4. It turns out, however, to be more convenient to work in the fixed space $\mathscr{L}_{\lambda}$. In particular the Heisenberg equations are in a sense equations obtained by taking covariant derivatives of operators in the bundle $\left\{\mathscr{L}_{\lambda}\right\}_{\lambda \in \Gamma}$ of Hilbert spaces over the coadjoint orbit $\Gamma$.

We are now in a position to formulate the main result of this section. In what follows $\left\langle\langle\cdot\rangle_{n, \lambda}\right.$ [see (4.20)] is considered to be a linear operator, which maps bounded operators in $\mathscr{H}_{\pi_{n}}$ into bounded operators in $L^{2}\left(\mathbb{R}^{v}, d^{v} x\right)$. Similarly $\langle\cdot\rangle_{\lambda}$ maps bounded operators in $\mathscr{L}_{\lambda}$ into bounded operators in $L^{2}\left(\mathbb{R}^{v}, d^{v} x\right)$.

Theorem 6.1. For any $(r, s) \in \mathbb{R}^{2 v}, a \in g$ and $|t|<\hat{T} \leqq T(\hat{T}>0$ depending on the initial conditions only) 


$$
\begin{aligned}
& s-\lim _{\substack{\hbar \rightarrow 0 \\
n \hbar=1}}\left\langle\left\langle U\left(\hbar^{-1 / 2} \alpha\right)^{*} U_{\hbar}(t)^{*} \exp \operatorname{ir}\left(q-\hbar^{-1 / 2} \xi^{t}\right)+i s\left(p-\hbar^{-1 / 2} \pi^{t}\right)\right.\right. \\
& \left.\left.\cdot \exp \hbar^{-1 / 2}\left(\pi_{n}\left(\frac{a}{n}\right)-\hat{\lambda}^{t}(a)\right) U_{\hbar}(t) U\left(\hbar^{-1 / 2} \alpha\right)\right\rangle\right\rangle_{n, \lambda} \\
& =\left\langle\left\langle\exp i\left(r q(t)+s p(t)+\Phi_{\lambda}(t)\left(\left(\operatorname{Ad} \gamma^{t}\right)^{-1} a\right)\right)\right\rangle\right\rangle_{\lambda}, \\
& s-\lim _{\substack{h \rightarrow 0 \\
n \hbar=1}}\left\langle\left\langle U\left(\hbar^{-1 / 2} \alpha\right)^{*} U_{\hbar}(t)^{*} \exp i\left(r q_{\hbar}+s p_{\hbar}\right) \exp \pi_{n}\left(\frac{a}{n}\right) U_{\hbar}(t) U\left(\hbar^{-1 / 2} \alpha\right)\right\rangle\right\rangle_{n, \lambda} \\
& =\exp i\left(r \xi^{t}+s \pi^{t}+\lambda^{t}(a)\right) .
\end{aligned}
$$

Proof. As in [H], (6.16) is an easy consequence of Relation (6.15), so we introduce

$$
W_{h}(t, s)=U\left(\hbar^{-1 / 2} \alpha^{t}\right)^{*} \pi_{n}\left(\gamma^{t}\right)^{*} U_{\hbar}(t-s) \pi_{n}\left(\gamma^{s}\right) U\left(\hbar^{-1 / 2} \alpha^{s}\right) \exp \frac{i}{\hbar} \int_{s}^{t} H_{c l}^{\prime}(r) d r,
$$

where

$$
H_{c l}^{\prime}(r)=H_{c l}\left(\xi^{r}, \pi^{r}, \lambda^{r}\right)-\lambda^{r}\left(\gamma^{r} \frac{d}{d r}\left(\gamma^{r}\right)^{-1}\right) .
$$

Then the expression on the left hand side of (6.15) may be written as

$$
\left.\left\langle W_{\hbar}(t, 0)^{*} \exp i \Phi_{n, \lambda}\left(\left(\operatorname{Ad} \lambda^{t}\right)^{-1} a\right)\right) \exp i(r q+s p) W_{\hbar}(t, 0)\right\rangle_{n, \lambda} .
$$

We will need another set of unitary operators, given in analogy to $\hat{W}[\operatorname{see}(6.10)]$ as

$$
\hat{W}_{\hbar}(t, s)=T \exp -i \int_{s}^{t} H_{f l}^{n}(r) d r
$$

on $\mathscr{H}_{\pi_{n}}$. Since for fixed $n,\left\|\pi_{n}(a)\right\| \leqq n\|a\|$ by (4.2), the existence of these operators follows as in $[\mathrm{H}]$ using a Dyson series on a suitable dense set and extending. In particular $\hat{W}_{\hbar}(t, s)\left|\psi_{x_{0}}\right\rangle$ is strongly differentiable in $t$ where $\left|\psi_{x_{0}}\right\rangle$ is of the form

$$
\psi_{x_{0}}(x)=\pi^{-v / 2} \exp -\left(x-x_{0}\right)^{2} / 2 \otimes \psi \quad\left(\psi \in h_{n}\right)
$$

which form a dense set. Also $\hat{W}_{h}(t, s)\left|\psi_{x_{0}}\right\rangle$ is in the domain of any polynomial in the $q_{i}, p_{j}$, and $\Phi_{n, \lambda}(a)(a \in g)$. Our first aim is to replace by $W_{\hbar}$ by $W_{\hbar}$ in (6.19) when considering matrix elements between states of the form $\phi_{x_{0}^{\prime}}$ and $\phi_{x_{0}}$ with

$$
\phi_{x_{0}}(x)=\pi^{-v / 2} \exp -\left(x-x_{0}\right)^{2} / 2 .
$$

To estimate the resulting error, consider

$$
\begin{aligned}
\left(W_{\hbar}(t, 0)-\hat{W}_{\hbar}(t, 0)\right)\left|\psi_{x_{0}}\right\rangle & =\int_{0}^{t} \frac{d}{d s}\left(W_{\hbar}(t, s) \hat{W}_{\hbar}(s, 0)\right)\left|\psi_{x_{0}}\right\rangle d s \\
& =i \int_{0}^{t} W_{\hbar}(t, s) \Delta^{s} \hat{W}_{\hbar}(s, 0)\left|\psi_{x_{0}}\right\rangle d s
\end{aligned}
$$

with

$$
\Delta^{s}=\hbar^{-1 / 2} H_{\hbar}^{2}(s)+Q_{1}(s)+Q_{2}(s)-H_{f l}^{n}(s)-\hbar^{-1} H_{c l}^{\prime}(s)
$$


Here

$$
\begin{gathered}
Q_{1}(s)=\frac{1}{i} \pi_{n}\left(\left(\gamma^{s}\right)^{-1}\right) \frac{d}{d s} \pi_{n}\left(\gamma^{s}\right)=i \pi_{n}\left(\left(\operatorname{Ad} \gamma^{s}\right)^{-1}\left(\gamma^{s} \frac{d}{d s}\left(\gamma^{s}\right)^{-1}\right)\right), \\
Q_{2}(s)=U\left(\hbar^{-1 / 2} \alpha^{s}\right)^{*} \frac{1}{i} \frac{d}{d s} U\left(\hbar^{-1 / 2} \alpha^{s}\right)=\hbar^{-1 / 2}\left(\frac{d}{d s} \pi_{j}^{s} q_{j}-\frac{d}{d s} \xi_{j}^{s} p_{j}\right) .
\end{gathered}
$$

Hence, by (6.8), (6.9), and (6.18), we obtain the estimate

$$
\left\|\left(W_{\hbar}(t, 0)-\hat{W}_{\hbar}(t, 0)\left|\psi_{x_{0}}\right\rangle\left\|\leqq \hbar^{1 / 2} \sup _{0 \leqq s \leqq t}\right\| B^{s} \hat{W}_{\hbar}(s, 0)\left|\psi_{x_{0}}\right\rangle \| .\right.\right.
$$

We are interested in obtaining a bound of (6.27) which is uniform in $\hbar$, namely for the special case $\psi_{x_{0}}=\psi_{x_{0}, g}^{n}$ with

$$
\psi_{x_{0}, g}^{n}(x)=\pi^{-v / 2} \exp -\left(x-x_{0}\right)^{2} / 2 \otimes \pi_{n}(g) \psi_{n \hat{l}_{0}},
$$

where $\psi_{n \hat{l}_{0}}$ is as in Sect. 4 and $g \in G$ satisfies

$$
\operatorname{Ad}^{*} g \hat{l}_{0}=\hat{\lambda} .
$$

Note that $P_{n}(\hat{\lambda})$ is the projection into the one-dimensional space spanned by $\pi_{n}(g) \psi_{n \hat{l}_{0}}$. To obtain the desired bound, note first that with the notation employed in (6.11) $H_{f l}^{n}(t)$ may be written as

$$
H_{f l}^{n}(t)=\sum_{i j} A_{i j}(t) X_{i}(n) X_{j}(n),
$$

where the $n$-independent, complex valued functions $A_{i j}(t)$ satisfy

$$
\sup _{\substack{|t| \leq T \\ i, j}}\left|A_{i j}(t)\right|<C
$$

for some $C<\infty$ uniformly in $n$. Now the Dyson series for $W_{\hat{n}}(s, 0)$ takes for form

$$
W_{h}(s, 0)\left|\psi_{x_{0}, g}^{n}\right\rangle=\sum_{m=0}^{\infty}(-i)^{m} \int_{0}^{s} \int_{0}^{s_{1}} \ldots \int_{0}^{s_{m-1}} d s_{m} \ldots d s_{1} H_{f l}^{n}\left(s_{1}\right) \ldots H_{f l}^{n}\left(s_{m}\right)\left|\psi_{x_{0}, g}^{n}\right\rangle .
$$

Therefore (6.11), (6.30), Lemma 4.7 and the analogous harmonic oscillator estimates for expectations of monomials in the $q_{i}$ and $p_{j}$ in the state $\phi_{x_{0}}$ give

$$
\| B^{s} \hat{W}_{\hbar}(s, 0)\left|\psi_{x_{0}, g}^{n}\right\rangle\left\|\leqq \sum_{m} \frac{|s|^{m}}{m !}(C)^{m+1}(2 m+4)^{m+2}\right\|\left|\psi_{x_{0}, g}^{n}\right\rangle \|
$$

for some new $C>0$ independent of $n$. Now the right hand side of (6.32) is absolutely convergent for all $|s| \leqq \hat{T} \leqq T(\hat{T}>0$ independent of $n)$ by Stirlings formula. Combining this with (6.26) we obtain

$$
\lim _{\substack{h \rightarrow 0 \\ n \hbar=1}} \hbar^{1 / 2} \|\left(W_{\hbar}(t, 0)-\hat{W}_{\hbar}(t, 0)\right)\left|\psi_{x_{0}, g}^{n}\right\rangle \|=0
$$

for all $|t| \leqq \hat{T}$. As a consequence, to prove (6.15), we may indeed replace $W_{\hbar}(t, 0)$ by $\hat{W}_{h}(t, 0)$ in $(6.19)$ and look at the resulting matrix elements between any states $\phi_{x_{0}^{\prime}}$ and $\phi_{x_{0}}$ (6.22). We recall that norm boundedness and weak convergence on a dense set implies strong convergence. Hence we have to show that 


$$
\begin{aligned}
F(n, t)= & \left\langle\psi_{x_{0}^{\prime}, g}^{n}\left|\hat{W}_{h}(t, 0)^{*} \exp i\left(r q+s p+\Phi_{n, \lambda}\left(\left(\operatorname{Ad} \gamma^{t}\right)^{-1} a\right)\right) \hat{W}_{\hbar}(t, 0)\right| \psi_{x_{0}, g}^{n}\right\rangle \\
& -\left\langle\phi_{x_{0}^{\prime}}\left|\left\langle\hat{W}(t, 0)^{*} \exp i\left(r q+s p+\Phi_{\lambda}\left(\left(\operatorname{Ad} \gamma^{t}\right)^{-1} a\right)\right) \hat{W}(t, 0)\right\rangle_{\lambda}\right| \phi_{x_{0}}\right\rangle .
\end{aligned}
$$

Satisfies

$$
\operatorname{Lim}_{n \rightarrow \infty}|F(n, t)|=0
$$

for all $|t| \leqq \hat{T}$. To see this, we expand both terms by writing down the Dyson series for $\hat{W}_{\hbar}(t, 0)$ and $\hat{W}(t, 0)$ respectively. Again by Lemma 4.7 and harmonic oscillator estimates for monomials in the $q_{i}$ and $p_{j}$ these series are uniformly convergent in $n$. If we combine the corresponding terms of each order, their difference vanishes as $n \rightarrow \infty$ by Lemma 4.5 . Hence $(6.35)$ follows by the Lebesgue dominated convergence theorem. This concludes the proof of Theorem 6.1 .

\section{Appendix A}

In this Appendix we give a proof of Lemma 5.2. Choose some large $R, K>0$, (they will be specified more below) and let

$$
F(\alpha, t)=\exp \left[-\int_{0}^{1}\left(i \pi_{n}\left(\frac{1}{n} a_{0, s}\right)+v_{s}\right) d s+\int \sum_{j=1}^{v} \pi_{n}\left(\frac{1}{n} a_{j, s}\right) d \alpha_{j}(s)\right] .
$$

Then

$$
\begin{aligned}
& d_{n}^{-1}\left|\int d^{v} x \operatorname{trace}_{h_{n}}\left\{E_{\alpha}(F(\alpha, t))-\exp -\beta\left(V(x)+i \pi_{n}\left(\frac{1}{n} A_{0}(x)\right)\right)\right\}\right| \\
& \leqq \\
& c_{1} \int_{|x| \leqq K} d^{v} x E_{\alpha}\left(\chi_{R}(\alpha)\left\|F(\alpha, t)-\exp -\beta\left(V(x)+i \pi_{n}\left(\frac{1}{n} A_{0}(x)\right)\right)\right\|\right) \\
& \quad+c_{1} \int_{|x|>K} d^{v} x E_{\alpha}\left(\chi_{R}(\alpha)\|F(\alpha, t)\|\right) \\
& \quad+c_{1} \int d^{v} x E_{\alpha}\left(\left(1-\chi_{R}\right)(\alpha)\|F(\alpha, t)\|\right) \\
& \quad+c_{1} \int_{|x|>K} d^{v} x e^{-\beta V(x)} \\
& \quad+c_{1} E_{\alpha}\left(\left(1-\chi_{R}\right)(\alpha)\right) \\
& =\mathrm{I}+\mathrm{II}+\mathrm{III}+\mathrm{IV}+\mathrm{V} .
\end{aligned}
$$

Here $\chi_{R}(\alpha)$ is the characteristic function of the set $\left\{\alpha\|\alpha\|_{\infty} \leqq R\right\}$ and we have used assumption (3.3) and (4.2). We first estimate the expectation in I. The estimate

$$
\left|e^{\alpha}-e^{\beta}\right| \leqq e^{\alpha}|\alpha-\beta| e^{|\alpha-\beta|}
$$

for real numbers $\alpha$ and $\beta$ and

$$
\left\|e^{A}-e^{B}\right\| \leqq\|A-B\| e^{\|A\|+\|B\|}
$$

for matrices $A, B$ combined with estimates (4.2) and our assumptions on the $A_{\mu}$ give 


$$
\begin{aligned}
& E_{\alpha}\left(\chi_{R}(\alpha)\left\|F(\alpha, t)-\exp -\beta\left(V(x)+i \pi_{n}\left(\frac{1}{n} A_{0}(x)\right)\right)\right\|\right) \\
& \quad \leqq c_{2} \exp -\beta V(x) E_{\alpha}\left(\chi_{R}(\alpha)\left(B_{1}+B_{2}+B_{3}\right) \exp \left(B_{1}+B_{3}\right)\right)
\end{aligned}
$$

where

$$
\begin{gathered}
B_{1}=\left|\int_{0}^{1}\left(v_{s}-\beta V(x)\right) d s\right|, \\
B_{2}=\left\|\int_{0}^{1}\left(a_{0, s}-\beta A_{0}(x)\right) d s\right\|, \\
B_{3}=\|\| \int_{0}^{1} \sum_{j=1}^{v} a_{j, s} d \alpha_{j}(s) \|
\end{gathered}
$$

depend on $\alpha$. Note that

$$
B_{2}<c_{3}
$$

uniformly in $\alpha, x$, and $t=n^{-2} \beta m^{-1}$. We now use Hölder's and Jensen's inequality to estimate the stochastic integral. This leads to a bound of the expression (A.5) in terms of $L^{2}(D \alpha)$-norms in the form

$$
\begin{aligned}
& c_{4} \exp -\beta V(x)\left(\left(\left\|\chi_{R} B_{1}\right\|_{2}+\left\|\chi_{R} B_{2}\right\|_{2}+\left\|\chi_{R} B_{3}\right\|_{2}\right)\right. \\
& \left.\quad \cdot \exp \left(\left\|\chi_{R} B_{1}\right\|_{2}+\left\|\chi_{R} B_{3}\right\|_{2}\right)\right) .
\end{aligned}
$$

Since $|x| \leqq K$, by the continuity of $V(x),\left\|\chi_{R} B_{1}\right\|_{2}$ is finite for all $t$ and $R$. Also $B_{1} \rightarrow 0$ a.s. as $t \rightarrow 0$. Therefore by the Lebesgue dominated convergence theorem $\left\|\chi_{R} B_{1}\right\|_{2}$ $<\operatorname{Min}(1, \varepsilon / 15 c)$ with

$$
c=e^{2} c_{4} \int \exp -\beta V(x) d^{v} x,
$$

provided $t \leqq t(R, K, \varepsilon)$. The same applies to $\left\|\chi_{R} B_{2}\right\|_{2}$ yielding another $\operatorname{Min}(1, \varepsilon / 15 c)$. Now consider the term $\left\|\chi_{R} B_{3}\right\|_{2}$. Using the relation

$$
\begin{gathered}
\sum_{j=1}^{v} \int_{0}^{1} A_{j}(x) d \alpha_{j}(s)=0 \\
\left\|\chi_{R} B_{3}\right\|_{2} \leqq
\end{gathered}
$$

by a standard bound for the $\alpha$-integral (see e.g. [Si2, p. 159]). Since $A_{j}$ is in $C_{0}^{\infty}$ we may apply the mean value theorem which yields the bound

$$
\begin{aligned}
c_{6} \sqrt{t}\left\{\left(\int_{0}^{1} d s\|\alpha(s)\|_{2}^{2}\right)^{1 / 2}+\int \frac{d s}{1-s}\left\|\alpha_{s}^{2}\right\|_{2}\right\} & \leqq c_{7} \sqrt{t}\left\{\left(\int_{0}^{1} d s s(1-s)\right)^{1 / 2}+\int_{0}^{1} \frac{d s}{1-s} s(1-s)\right\} \\
& \leqq \operatorname{Min}(1, \varepsilon / 15 c)
\end{aligned}
$$

for $t \leqq t(\varepsilon)$. This gives a bound $\varepsilon / 5$ for the term I in (A.2) provided $t \leqq t(R, K, \varepsilon)$. 
Next we estimate the term III in (A.2).

$$
\begin{aligned}
\mathrm{III} & =c_{1} \int d^{v} x E_{\alpha}\left(\chi\left(\alpha,\|\alpha\|_{\infty} \geqq R\right)\|F(\alpha, t)\|\right) \\
& \leqq c_{8} \int d^{v} x E_{\alpha}\left(\chi\left(\alpha,\|\alpha\|_{\infty} \geqq R\right) \exp \left[-\int_{0}^{1} v_{s} d s\right]\right) \\
& =c_{8} \int d^{v} x E_{\alpha}\left(\chi\left(\alpha,\|\alpha\|_{\infty} \geqq R\right) \exp \left[-\beta \int_{0}^{1} V(x+\sqrt{t} \alpha(s)) d s\right]\right) \\
& =c_{8} E_{\alpha}\left(\chi\left(\alpha,\|\alpha\|_{\infty} \geqq R\right)\right) \int e^{-\beta V(x)} d^{v} x .
\end{aligned}
$$

In the last equality we have used Fubini's theorem. Hence

$$
\mathrm{III} \leqq c_{9} \frac{1}{R} E_{\alpha}\left(\sup _{s \in[0,1]}|\alpha(s)|\right) \leqq c_{10} \frac{1}{R}
$$

where the last estimate follows e.g. from [Po], Theorem II.2. Therefore

$$
\mathrm{III}+\mathrm{V} \leqq 2 \cdot \varepsilon / 5
$$

provided $R \geqq R(\varepsilon)$.

We turn to a discussion of the term II.

$$
\begin{aligned}
\mathrm{II} & =c_{1} \int_{|x|>K} d^{v} x E\left(\chi_{R}(\alpha)\|F(\alpha, t)\|\right) \\
& \leqq c_{8} \int_{|x|>K} d^{v} x E_{\alpha}\left(\chi_{R}(\alpha) \exp \left[-\beta \int_{0}^{1} V(x+\sqrt{t} \alpha(s)) d s\right]\right) \\
& \leqq c_{8}\left[\int d^{v} x E_{\alpha}\left(\exp \left[-\beta \int_{0}^{1} V(x+\sqrt{t} \alpha(s)) d s\right]\right)\right. \\
& \left.-\int_{|x|<K} d^{v} x E_{\alpha}\left(\chi_{R}(\alpha) \exp \left[-\beta \int_{0}^{1} V(x+\sqrt{t} \alpha(s)) d s\right]\right)\right] .
\end{aligned}
$$

Now set

$$
V_{-}(x, \sqrt{t} R)=\operatorname{Min}_{|y| \leqq R \sqrt{t}} V(x+y) .
$$

Then we obtain

$$
\mathrm{II} \leqq c_{7}\left[\int d^{v} x E_{\alpha}\left(\exp \left[-\beta \int_{0}^{1} V(x+\sqrt{t} \alpha(s)) d s\right]\right)-\int_{|x|<: z} e^{-\beta V_{-}(x, \sqrt{t} R)} d^{v} x E_{\alpha}\left(\chi_{R}\right)\right] .
$$

Now by [Si2, Theorem 10.1] the first term in the right-hand side of (A.18) converges to $\int d^{v} x e^{-\beta V(x)}$ as $t \rightarrow 0$ and by the preceding considerations $E_{\alpha}\left(\chi_{R}\right) \rightarrow 1$ as $R \rightarrow \infty$. Hence for all $t \leqq t(\varepsilon)$ and $R \geqq R(\varepsilon)$

$$
\mathrm{II} \leqq c_{7}\left\{\int d^{v} x e^{-\beta V(x)}-\int_{|x| \leqq K} e^{-\beta V-(x, \sqrt{t} R}\right\}+\frac{\varepsilon}{10} .
$$

Now for fixed $R \quad V_{-}(x, \sqrt{t} R) \rightarrow V(x)$ as $t \rightarrow 0$ by continuity for all $|x| \leqq K$. By dominated convergence $\left(\left|V_{-}\right| \leqq|V(x)|\right)$ the second term in (A.19) converges to 
$\int_{|x| \leqq K} e^{-\beta V(x)} d^{v} x$. Therefore, if first $R$ is chosen large, then $K$ large and then $t$ small, we obtain

Also

$$
\mathrm{II} \leqq \frac{\varepsilon}{5}
$$

for all $K \geqq K(\varepsilon)$.

$$
\mathrm{IV} \leqq \frac{\varepsilon}{5}
$$

Combined we have

$$
\mathrm{I}+\mathrm{II}+\mathrm{III}+\mathrm{IV}+\mathrm{V} \leqq \varepsilon
$$

concluding the proof of the lemma.

Acknowledgement. One of the authors (R.S.) would like to thank C. N. Yang for his hospitality at the Institute for Theoretical Physics at SUNY, Stony Brook.

\section{References}

[BSSW] Balachandran, A.P., Salomonson, P., Skagerstam, B.S., Winneberg, J.O.: Classical description of a particle interacting with a non-abelian gauge field. Phys. Rev. D15, 2308-2317 (1977)

[BCL] Barducci, A., Casalbuoni, R., Lusanna, L.: Classical spinning particles interacting with external gravitational field. Nucl. Phys. B124, 521-538 (1977)

[BW] Brown, S.L., Weisberger, W.I.: Vacuum polarızation in uniform non-abelian gauge fields. Nucl. Phys. B 157, 285-326 (1979)

[CSS] Combes, J., Schrader, R., Seiler, R.: Classical bounds and limits for energy distributions of Hamilton operators in electromagnetic fields. Ann. Phys. 111, 1-18 (1978)

[CKS] Cotta-Ramusino, P., Krüger, W., Schrader, R.: Quantum scattering by external metrics and Yang-Mills potentials. Ann. Inst. H. Poincare, 31, 43-71 (1979)

[DC] Dresden, M., Chen, S.F.: Solitons, gauge theories and the "great Einstein theorem". Physica 83 A, 1-17 (1975)

[E] Ehrenfest, P.: Bemerkung über die angenäherte Gültigkeit der klassischen Mechanik innerhalb der Quantenmechanik. Z. Physik 45, 455-457 (1927)

[FL] Fuller, W., Lenard, A.: Generalized quantum spins, coherent states, and Lieb inequalities. Commun. Math. Phys. 67, 69-84 (1979)

[G] Gilmore, R.: The classical limit of quantum nonspin systems. J. Math. Phys. 20, 891-893 (1979)

[Go] Golden, S. : Lower bounds for the Helmholtz function. Phys. Rev. 137B, 1127-1128 (1965)

[GS] Guillemin, V., Sternberg, S.: On the equations of motion of a classical particle in a YangMills field and the principle of general covariance. Hadronic J. 1, 1-32 (1978)

[H] Hepp, K.: The classical limit for quantum mechanical correlation functions. Commun. Math. Phys. 35, 265-277 (1974)

[HSU] Hess, H., Schrader, R., Uhlenbrock, D.A.: Domination of semigroups and generalization of Kato's inequality. Duke Math. J. 44, 893-904 (1977)

[Ho] Hogreve, H.: Freie Universität Berlin, Thesis (unpublished) (1983)

[K] Kato, T.: In: Topics in analysis, pp. 185-195. London, New York: Academic Press 1978

[Ki] Kirillov, A.A.: Elements of the theory of representations. Berlin, Heidelberg, New York: Springer 1976

[K1] Klauder, J.R.: The action option and a Feynman quantization of spinor fields in terms of ordinary C-numbers. Ann. Phys. 11, 123-168 (1960)

[Ko] Kostant, B.: In: Lecture Notes in Mathematics, Vol. 170. Berlin, Heidelberg, New York: Springer 1970 
[L] Lieb, E.: The classical limit of quantum spin systems. Commun. Math. Phys. 31, 327-340 (1973)

[P] Perelomov, S.: Coherent states for arbitrary Lie group. Commun. Math. Phys. 26, 222-236 (1979)

[Po] Potthoff, J.: Bielefeld Preprint BIT P82/10, 1982. J. Math. Phys. (to appear)

[Sch] Schechter, J.: Yang-Mills particle in 't Hooft's gauge field. Phys. Rev. D14, 524-527 (1976)

[St] Schrader, R., Taylor, M.: In preparation

[Si1] Simon, B.: The classical limit of quantum partition functions. Commun. Math. Phys. 71, 247-276 (1980)

[Si2] Simon, B.: Functional integration and quantum physics. London, New York: Academic Press 1979

[Si3] Simon, B.: Abstract Kato's inequality for generators of positivity preserving semigroups. Ind. Math. J. 26, 1067-1073 (1977)

[Sou] Souriau, J.M.: Structures des systemes dynamiques. Paris: Dunod 1972

[S] Stern, A.: Resonances in SU(2) gauge theory. Phys. Rev. D15, 3672-3674 (1977)

[St] Sternberg, S.: Minimal coupling and the symplectic mechanics of a classical particle in the presence of a Yang-Mills field. Proc. Natl. Acad. Sci. USA 74, 5253-5254 (1977)

[Sy] Symanzik, K.: Proof and refinements of an inequality of Feynman. J. Math. Phys. 6, $1155-1156$ (1965)

[T1] Thompson, C.: Inequality with applications in statistical mechanics. J. Math. Phys. 6, $1812-1813$ (1965)

[T2] Thompson, C.: Inequalitites and partial orders on matrix spaces. Ind. Math. J. 21, 469-480 (1971)

[W] Wong, S.K.: Field and particle equations for the classical Yang-Mills field and particles with isotropic spin. Nuovo Cimento 65 A, 689-694 (1970)

Communicated by A. Jaffe

Received July 7, 1983

Note added in proof. The basic relation (4.1) has also been derived and used by D. Zelobenko (Compact Lie groups and their representations. Transl. Math. Monogr. Providence, RI: AMS 1973).

In $[\mathrm{St}]$ it will be shown that the formal series in $\hbar$ for the partition function as derived by Uhlenbeck, Gropper, and Wigner is indeed an asymptotic expansion. 